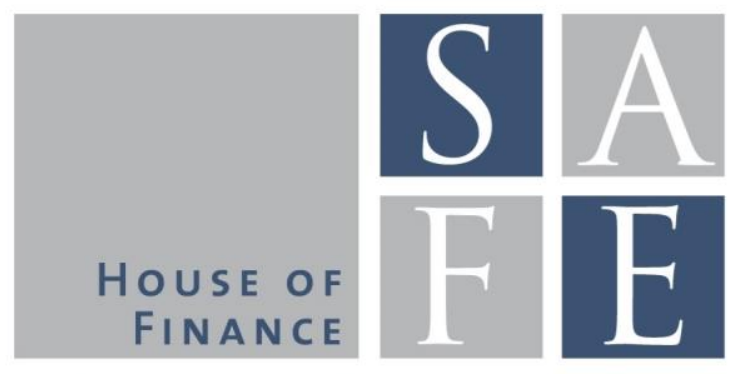

Working PAPER SERIES

Sascha Baghestanian - Todd B. Walker

\title{
Anchoring in Experimental Asset Markets
}

SAFE Working Paper No. 54

SAFE I Sustainable Architecture for Finance in Europe

A cooperation of the Center for Financial Studies and Goethe University Frankfurt 


\section{Non-Technical Summary}

Markets play an important role in the aggregation of diverse information. Competitive markets coordinate heterogeneous relative evaluations of goods and services produced by society via transaction prices. While individual evaluations of participants in a market place are usually unobservable, competitive market prices provide signals about the aggregate fundamental value of a traded item. Whenever a market satisfies the property that transaction prices reflect fundamentals perfectly, economists refer to it as efficient or as having the price-discovery property. In an efficient market high prices indicate that society values a good highly, potentially fostering its supply and reducing its demand.

However, ex-ante there is no guarantee that markets fulfill their important role to accelerate pricediscovery; maybe not even approximately. If mis-pricing on a market is persistently positive, i.e. if prices are above the fundamental value for an extended period of time, economists usually speak of a bubble. Bubbles are not only characterized by long periods of over-pricing but are also (at least empirically) usually associated with a significant crash of prices towards the fundamental value. As Kindleberger and Aliber (2005) put it: "Bubbles always implode". The consequences of bubblecrash patterns, independently of the market, are usually severe. Bubbles lead to over-investment and mis-allocation of capital since the periods of increasing prices sent the wrong signal to producers. The crash, on the other hand, renders these investments unprofitable. Put differently, bubble-crash patterns in specific markets can lead to a redistribution of wealth and social turmoil which may affect the rest of the economy.

There are numerous examples for bubble-crash episodes, some of which are mentioned here. They range from the Dutch tulip-mania (1634-1637), the US-stock mania (1928-1932), the dot-com bubble (1998-2001), the uranium bubble (2004 -2008) and, most recently, the US real estate bubble (1996-2009). Although there is suggestive evidence indicating that the aforementioned episodes faced a bubble, there is no guarantee that these markets were mis-priced since the underlying fundamental values are not observable.

One way to circumvent the problem of unobservable fundamental values and to study the causes and the cures of bubbles and crashes is to utilize experimental methods. Experimental economists since Vernon Smith et al. (1988) have focused their attention on mis-pricing in experimental asset markets. Smith and his co-authors illustrate that asset prices may deviate systematically from their underlying fundamental value (FV) even in controlled laboratory environments in which the dividend distribution is common knowledge. Moreover, they show that experimental asset prices follow bubble-crash dynamics: Initially, asset prices increase beyond the fundamental value until they peak and "crash" back towards the FV. The observed price-dynamic proved to be highly replicable and persist under various experimental treatments. 
Our work uses experimental methods to investigate one particular cause for the formation of bubbles in laboratory environments: visual stimuli. We show that visual stimuli induce anchoring behavior (Kahneman and Tversky $(1983,1974))$ that can substantially mitigate bubble behavior. We set visual stimuli by manipulating within-period price charts used by our experimental traders. Most importantly, our evidence suggests that the visual stimulus needs to be provided only in the first period of a standard asset market experiment to affect overall price dynamics. We support our hypothesis with new experimental evidence from 22 laboratory sessions (216 subjects) and adapt existing theoretical frameworks to rationalize our findings.

Our insights suggest that trading behavior in the initial period is crucial for generating the wellestablished bubble-crash dynamics in experimental asset markets. Inducing an anchor at the fundamental value in the first period is sufficient to eliminate or significantly reduce bubbles in laboratory environments. If no anchor is set, standard bubble-crash patterns emerge.

Our insights further improve our understanding of stock market dynamics and suggest that setting initial prices is perhaps more important than previously believed. Stock Exchanges such as the New York Stock Exchange (NYSE) determine opening prices through pre-opening auctions. Between 8.00am and 9.30am, market makers at the NYSE collect limit orders and try to implement a market clearing price. Our findings suggest that opening prices have very important implications for subsequent intra-day price dynamics. More specifically, underpricing the asset during the preopening may induce severe price rallies peaking well above the assets fundamental value. Our work therefore suggests that the market structure and the price determination in pre-openings should be discussed when addressing asset market stability.

The results presented in our paper also contribute to the well-established literature on initial public offerings (IPO). Interpreting the first-period price in our experimental sessions as the IPO price of a stock, our findings suggest that mis-pricing the IPO could lead to non-trivial price dynamics. Since our experimental setup gives us more control over the "IPO price" of the stock, we can make predictions about the consequences of over- and under-pricing the asset initially. 


\title{
Anchoring in Experimental Asset Markets*
}

\author{
Sascha Baghestanian ${ }^{\dagger}$ and Todd B. Walker:
}

10th February 2015

\begin{abstract}
We investigate the relationship between anchoring and the emergence of bubbles in experimental asset markets. We show that setting a visual anchor at the fundamental value $(\mathrm{FV})$ in the first period only is sufficient to eliminate or to significantly reduce bubbles in laboratory asset markets. If no $\mathrm{FV}$-anchor is set, bubble-crash patterns emerge. Our results indicate that bubbles in laboratory environments are primarily sparked in the first period. If prices are initiated around the FV, they stay close to the FV over the entire trading horizon. Our insights can be related to initial public offerings and the interaction between prices set on pre-opening markets and subsequent intra-day price dynamics.
\end{abstract}

Keywords: Experimental Asset Markets, Anchoring, Bubbles

JEL Classifications: C90, C91, D03, G02, G12

${ }^{*}$ We gratefully acknowledge research support from the Research Center SAFE, funded by the State of Hessen initiative for research LOEWE. This project benefited from many helpful comments and suggestions. We particularly thank Carey Deck, John Duffy, Martin Götz, Michael Kirchler, Michael Kosfeld, Baptiste Massenot, Thomas Mosk, Devesh Rustagi and Ferdinand v. Siemens. We also thank two anonymous referees as well as the editors for very helpful suggestions and advice.

${ }^{\dagger}$ Department of Economics, Goethe University, House of Finance, Grüneburgplatz 1, Rm 4.12, 60323 Frankfurt, Germany; e-mail:baghestanian@econ.uni-frankfurt.de

${ }^{\ddagger}$ Corresponding Author -- Department of Economics, Indiana University, Wylie Hall Rm 304,100 S. Woodlawn, Bloomington, IN 47405-7104; e-mail:walkertb@indiana.edu 


\section{Introduction}

In a seminal paper, Smith et al. [1988] (SSW) illustrate that asset prices may deviate systematically from the underlying fundamental value (FV) even in controlled laboratory environments. Moreover, SSW show that experimental asset prices follow bubble-crash dynamics: Initially, asset prices increase beyond the fundamental value until they peak and "crash" back towards the FV. ${ }^{1}$ The observed price-dynamics proved to be highly replicable and persist under various experimental treatments (King et al. [1993], Boening et al. [1993], Porter and Smith [1995], Caginalp et al. [1998], Smith et al. [2000], Caginalp et al. [2000], Dufwenberg et al. [2005], Noussair and Tucker [2006], Haruvy and Noussair [2006], Haruvy et al. [2007], Hussam et al. [2008], Williams [2008]).

We show that visual stimuli (i.e.: within-period price charts) induce anchoring behavior (Kahneman and Tversky $[1983,1974])$ that can substantially mitigate experimental asset price bubbles. Most importantly, our evidence suggests that the visual stimulus needs to be provided in the first period only to affect overall price dynamics. We support our hypothesis with new experimental evidence from 22 laboratory sessions (216 subjects) and adapt existing theoretical frameworks to rationalize our findings (Caginalp et al. [2000], Duffy and Ünver [2006]).

Our insights suggest that trading behavior in the initial period is crucial for generating the well-established bubble-crash patterns in experimental asset markets. Our findings illustrate that actual restrictions of initial prices are not necessary to affect overall price dynamics (Caginalp et al. [2000]). Instead, simply inducing a visual anchor at the fundamental value in the first period is sufficient to eliminate or significantly reduce bubbles in laboratory environments. If no such anchor is set, standard bubble-crash patterns emerge, even if the fundamental value is constant over time (Noussair et al. [2001]). ${ }^{2}$

To further illustrate the importance of anchors in experimental asset markets, we present data on treatments in which first-period-anchors were set at "normatively irrelevant random numbers" (Ariely et al. [2003]). Setting anchors at values which exceed the fundamental value, induces price-paths which initiate around the anchor and which slowly converge towards the FV from above. Setting anchors at values which are significantly smaller than the FV induces price paths, which tend to initiate below the FV, over-shoot the fundamental value and crash back or converge to it towards the end of the trading horizon. We use the models of Duffy and Ünver [2006] and Caginalp et al. [2000] -incorporating our anchoring insights- to explain the observed asymmetry. ${ }^{3}$

\footnotetext{
${ }^{1}$ The resulting price paths therefore violate basic predictions associated with the homogeneous-beliefs, rational expectations equilibrium.

${ }^{2}$ In a slightly different context than ours, Caginalp et al. [2008] observe that subjects anchor their price expectations to previous prices or valuations, suggesting that anchoring may affect behavior in experimental asset markets.

${ }^{3}$ Caginalp et al. [2000] investigate initial price restrictions below the FV on the observed price dynamics. We extend this line of work by inducing initial prices both below and above the FV via visual stimuli instead of using actual restrictions and contribute by showing that the observed price-dynamics are asymmetric.
} 
Our insights further improve our understanding of stock market dynamics and suggest that setting initial prices is perhaps more important than previously believed. Stock Exchanges such as the New York Stock Exchange (NYSE) determine opening prices through pre-opening auctions. Between 8.00am to 9.30am, market makers at the NYSE collect limit orders and try to implement a market clearing price. ${ }^{4}$ Our findings suggest that opening prices have very important implications for subsequent intra-day price dynamics. However, investigating the impact of opening prices on intra-day price dynamics in field settings is difficult due to potential endogeneity concerns. Our laboratory environment provides more control over initial prices and allows us to make causal statements regarding the relationship between initial prices and subsequent price dynamics.

The results presented in this paper also contribute to the well established literature on initial public offerings (IPO's, Ritter and Welch [2002], Loughrand and Ritter [2002]). Interpreting the first-period price in our experimental sessions as the IPO price of a stock, our findings suggest that mis-pricing the IPO could lead to non-trivial price dynamics. Since our experimental setup gives us more control over the "IPO price" of the stock, we can make predictions about the consequences of over- and under-pricing the asset initially. ${ }^{5}$

Our findings are tightly related to a new emerging branch in the experimental asset market literature, which explores the relationship between visualization of trading prices and bubble formation. For instance, Cason and Samek [2014] illustrate that the provision of price-charts in standard asset market experiments mitigates bubbles. Powell [2010] presents an eye-tracking study and correlates behavior with visual attention in experimental asset markets. He observes patterns which further emphasize the importance of focus and visual attention on behavior in laboratory asset markets.

Our results also contribute to an ongoing debate on the impact of fundamental value processes on bubble formation (Kirchler et al. [2012], KHS hereafter). ${ }^{6}$ Our insights suggest that constant fundamental values, coupled with a visual representation of trading prices, might favor anchoring towards the FV.

The paper is organized as follows. Section 2 presents our experimental design. Section 3 presents our results. Section 4 applies the model of Duffy and Ünver [2006] to our data, showing that anchoring is an important driver of our observed treatment effects. Section 5 concludes the paper.

\footnotetext{
${ }^{4}$ Opening prices have been discussed extensively in the literature: See e.g.: Amihud and Mendelson [1987], Stoll and Whaley [1990], Biais et al. [1999] for field evidence and (e.g.) Biais et al. [2013] for experimental evidence.

${ }^{5}$ Our work is also connected to the literature on information revelation and asset price dynamics in experimental settings. Gillette et al. [1999] and Porter et al. [2010] find support for under-reaction to news on asset valuation.

${ }^{6} \mathrm{KHS}$ argue that "confusion" about the decreasing fundamental value process, coupled with an increasing cashto-asset value $(\mathrm{C} / \mathrm{A})$ ratio, are the most important factors generating the typical bubble-crash patterns in laboratory asset markets which use the SSW-design. Indeed, experiments intended to reduce this confusion by changing the notion of a "stock" to a "stock of a depletable gold mine," which served to instill the idea of a declining FV, mitigated bubble behavior.
} 


\section{Experimental Design}

In our laboratory environments participants traded a dividend-paying asset over a ten period trading horizon, using an experimental currency (Taler). Within each period a standard double auction trading mechanism determined bilateral trading prices. At the beginning of each experimental session half of the participants were endowed with 20 shares and 3,000 Taler. The other half received an initial endowment of 60 shares and 1,000 Taler.

Following the evidence of Caginalp et al. [1998, 2000], Noussair et al. [2001], Caginalp and Ilieva [2005], Haruvy and Noussair [2006] and Kirchler et al. [2012], we implemented an environment in which the fundamental value and the $\mathrm{C} / \mathrm{A}$ ratio are constant over time. That is, dividends took realizations of 5 and -5 "Taler" with equal probability of $50 \%$. Dividend-payments/losses were collected in a separate account to keep the $\mathrm{C} / \mathrm{A}$ ratio constant over time. The $\mathrm{FV}$ of the asset was kept constant at a value of 50 Taler over the 10 periods trading horizon, by ensuring a terminal buyback value of 50 .

Within each trading period we use price-charts to depict the history of transaction prices. Our within-period price plots share the same features as the price charts used by KHS.

To be specific, in the first period, price-charts initiate at the fundamental value of the asset. Hence, within the first trading period, if the first trading price is above (below) the FV, subjects observe a price path -connecting the initial value $(=50)$ to the new price $(>50$ or $<50)$ - which suggests that prices have "increased" ("decreased"). Moreover, after initial transactions are made in period one, and if new offers are made by subjects, the graph re-initiates the prices at the FV. Hence, in the first period, subjects observe price paths which "jump" back into the FV after new offers are posted (See Figures $5 \mathrm{a}$ and $5 \mathrm{~b}$ in Appendix A).

In the follow-up periods, the price plot initiates at the previous period's average price, and there is no re-initiation in the FV after transactions as in period one. However, the scale of the $y$-axis is not constant across trading periods: The maximum of the y-axis is a multiple of the previous period's transaction prices, potentially channeling/anchoring perceived prices towards more stable outcomes and affecting observed price dynamics.

We conjecture that the seemingly innocuous graphical interface features described above induce anchoring behavior and generate bubble mitigation effects. We further hypothesize that eliminating these design-features will have important effects on the observed price dynamics even if the $\mathrm{FV}$ and the $\mathrm{C} / \mathrm{A}$ ratio are constant over time. However, our study is not designed to identify the effect of every single particularity of the graphical interface. Our aim is simply to show that anchoring behavior can be an important aspect in experimental asset markets, in particular if FV's are constant.

We ran four treatments to test these conjectures and compare the price dynamics in terms of over-valuation -via the "relative deviation" (RD; Kirchler et al. [2010])- and in terms of mis-pricing 
-via the "relative absolute deviation" (RAD; Kirchler et al. [2010]).

First, we run a constant FV and constant C/A-ratio treatment in which we keep the y-axis for the within-period price chart fixed at a value of 250. In this treatment we initiate the first period price at the FV (50) and let prices jump back to the FV as outlined above. ${ }^{8}$ Furthermore, in the price-chart, prices were initiated in the FV (50) and jumped back into the FV after every transaction in the first period. We denote this treatment as "Anchor-T4 $(\backslash=)$ ". We will show below that this treatment generates trading prices, which mimic the trading prices of KHS. ${ }^{9}$ Treatment "Anchor- $\mathrm{T} 4(\backslash=)$ " is therefore our control treatment.

Second, we ran another treatment, denoted as "NoAnchor-T4 $(\backslash=)$ ", in which we did not initiate the real-time graphical representation of trading prices at the FV and we eliminate the re-initiation component in period one. That is, in the price-chart, prices did not jump back into the FV after transactions in the first period. The scale of the y-axis in the price chart was again fixed at 250. Hence, the only difference between treatments "NoAnchor-T4 $(\backslash=)$ " and "Anchor-T4( $\backslash=)$ " is the initiation of the price chart at the FV and the "jump-back" component in period one. ${ }^{10}$ We show below that both treatments differ significantly in terms of the associated price dynamics.

While treatments "NoAnchor-T4 $(\backslash=)$ " and "Anchor-T4 $(\backslash=)$ " illustrate the difference between asset market experiments in which we set an anchor around the FV and experiments in which we do not set an anchor, they do not tell us what happens if we would try to set an anchor at a normatively irrelevant number.

To investigate the latter question we ran two more treatments. First, we replicated treatment "Anchor-T4 $(\backslash=)$ " with the only difference that prices were initiated at 10 Taler and prices were allowed to jump back into the value of 10 in the first period. We denote this treatment as "Anchor10-T4 $(\backslash=)$ ". Second, we again replicated treatment "Anchor-T4 $(\backslash=)$ " with the only difference that prices were initiated at 90 Taler and prices were allowed to jump back into the value of 90 . We denote the latter treatment as "Anchor90-T4( $\backslash=)$ ". In both treatments the scale of the $\mathrm{y}$-axis in the price chart was again fixed at 250 .

For our experiments we recruited subjects via the ORSEE system at Goethe University, Frankfurt. We ran six sessions of treatment "NoAnchor-T4 $(\backslash=)$ ". In four of the six sessions, 10 subjects participated. In the two remaining sessions, nine and seven subjects participated. ${ }^{11}$ We also ran six sessions of treatment "Anchor-T4 $(\backslash=)$ ". In each of the six sessions, 10 subjects participated. Finally, we ran five sessions of treatment "Anchor10-T4 $(\backslash=)$ " and five sessions of treatment

\footnotetext{
${ }^{7} \mathrm{RAD}=\frac{1}{10} \sum_{t=1}^{10} \frac{\left|P_{t}-F V_{t}\right|}{\operatorname{mean}(F V)}, \mathrm{RD}=\frac{1}{10} \sum_{t=1}^{10} \frac{P_{t}-F V_{t}}{\operatorname{mean}(F V)}$ where $P_{t}$ denotes the average within period transaction price.

${ }^{8}$ This treatment is comparable to treatment $\mathrm{T} 4(\backslash=)$ in KHS with the main difference that the y-axis for the within-period price chart was fixed at 250. No other component was changed in the design of KHS.

${ }^{9}$ However, not that we are not replicating KHS. It might well be that the provision of an anchor is more important if the scale of the y-axis is fixed at (e.g.) 250 .

${ }^{10} \mathrm{We}$ argue that both factors together generate an anchor towards the FV and mitigate bubbles.

${ }^{11}$ The two sessions with the comparatively small number of participants actually show more stable price paths than the other treatments.
} 
Table 1: Experimental Design Parameters and Characteristics

\begin{tabular}{lccccc}
\hline Treatment & Sessions & Subjects & Unit Endowments & Cash Endowments & Trading Periods \\
\hline \hline & & & & & \\
"Anchor-T4 $(\backslash=)$ " & 6 & 56 & Half 60, Half 20 & Half 1000, Half 3000 & 10 \\
"NoAnchor-T4 $(\backslash=)$ " & 6 & 60 & Half 60, Half 20 & Half 1000, Half 3000 & 10 \\
"Anchor10-T4 $(\backslash=)$ " & 5 & 50 & Half 60, Half 20 & Half 1000, Half 3000 & 10 \\
"Anchor90-T4 $(\backslash=) "$ & 5 & 50 & Half 60, Half 20 & Half 1000, Half 3000 & 10 \\
\hline Overall & 22 & 216 & & & \\
Taler-Euro & & & & & \\
Conversion Rate & $400: 1$ & & & \\
Dividends & $\{-5,5\}(50 \%)$ & & & & \\
Buyback value at T & 50 & & & & \\
\hline
\end{tabular}

"Anchor90-T4 $(\backslash=)$ ". In each of the ten sessions, 10 subjects participated. The experimental design parameters and session-characteristics are summarized in Table $1 .{ }^{12}$

\section{Results}

Figures 1a and 1b show average transaction prices under treatment "NoAnchor-T4 $(\backslash=)$ " and treatment "Anchor-T4 $(\backslash=)$ " respectively. Session-specific transaction prices are shown in Figures 7a 7c in Appendix C. Note that our prices in the "NoAnchor-T4 $(\backslash=)$ "-treatment deviate substantially from the prices in our "Anchor-T4 $(\backslash=)$ " treatment. Prices under treatment "Anchor- $\mathrm{T} 4(\backslash=)$ ", mimic the prices observed by KHS.

We present session-specific RAD measures for our "NoAnchor"-treatment in the first column of Table 2. The corresponding RAD-measures of KHS, denoted as T4 $(\backslash=)$, are shown in column two. A Mann-Whitney test indicates that the median RAD in our "NoAnchor" sessions exceeds the median RAD measure of KHS significantly at a $5 \%$ level $(\mathrm{p}$-value $=0.041) .{ }^{13}$ The difference in RD-measures between our NoAnchor-treatment and $\mathrm{T} 4(\backslash=)$ is not significant (p-value $=0.1797$; columns 3 and 4). However, given the size of the sample it is questionable whether this "insignificance" is due to the power-properties of the test. If we eliminate, for instance, the lowest or second lowest RD measure from our sessions, we obtain a significant difference at a $10 \%$ level.

The RAD and RD measures for our "Anchor-T4( $\backslash=)$ "-treatment are shown in column 5 and 6 of Table 2. First, we observe that our control treatment generates significantly less mis-pricing in comparison to our "NoAnchor"-treatment, at least at a $10 \%$ level. ${ }^{14}$ This is surprising since the

\footnotetext{
${ }^{12}$ We use the same design-parameters, materials and procedures as KHS. We accessed the zTree-software used by the authors as well as their instructions via the AEA-database. We described the cash and unit endowments above. We used a Taler-Euro conversion rate of 400:1. Each session lasted approximately 90 minutes (including procedural overhead). Subjects earned on average around 15 Euros in every session (includes a 5 Euro show-up fee).

${ }^{13}$ Given the small sample sizes we applied exact p-values in our statistical analyses, using $\mathrm{R}$ as a software of our choice.

${ }^{14}$ Mann-Whitney test $p-$ value $=0.064$. Looking at Figures $7 \mathrm{a}-7 \mathrm{c}$, we observe that the differences in the price
} 


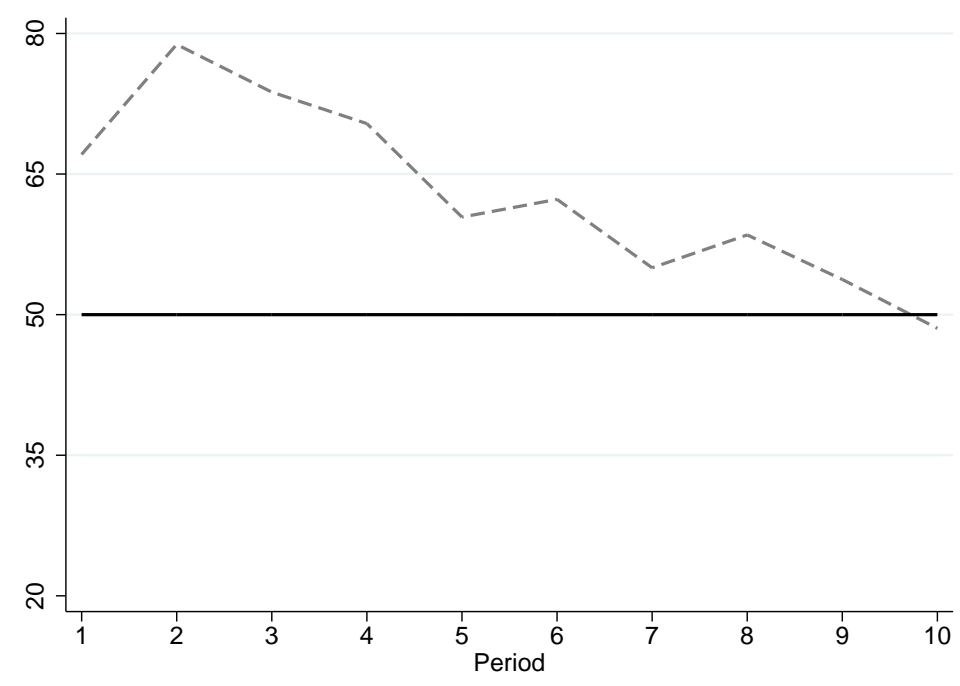

(a) Average Session Prices, "NoAnchor-T4( $\backslash=)$ ".

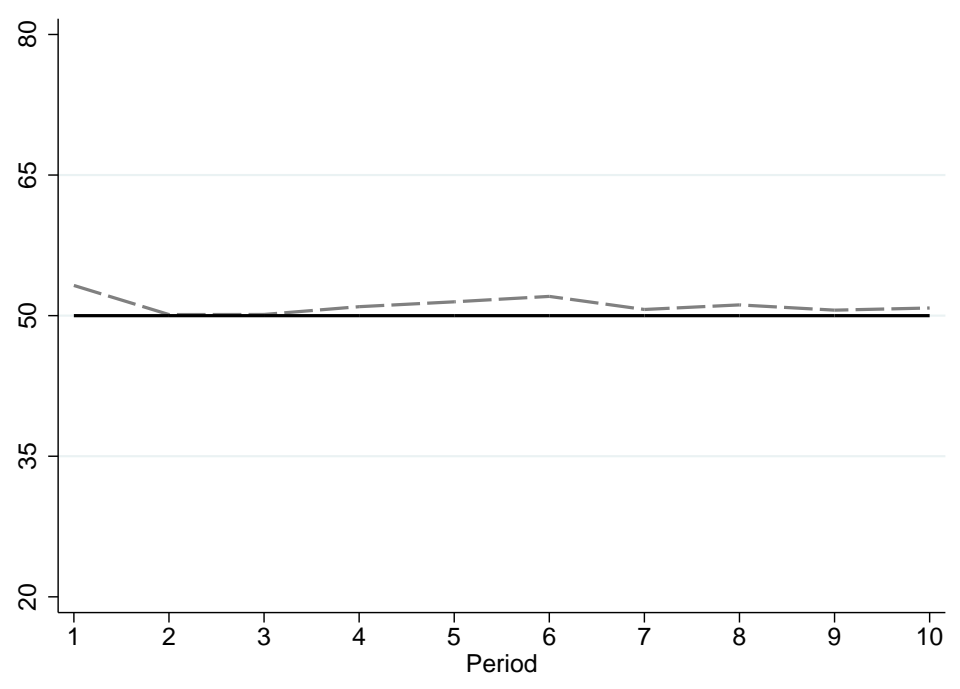

(b) Average Session Prices, "Anchor-T4( \=)".

Figure 1: Dashed Lines: Average Session Prices. Solid Line: FV.

\begin{tabular}{|c|c|c|c|c|c|}
\hline \multicolumn{2}{|l|}{ RAD } & \multicolumn{2}{|l|}{$\mathrm{RD}$} & \multirow{2}{*}{$\begin{array}{c}\text { RAD } \\
\text { "Anchor-T4 }(\backslash=) "\end{array}$} & \multirow{2}{*}{$\begin{array}{c}\mathrm{RD} \\
\text { "Anchor-T4( } \backslash=) \text { " }\end{array}$} \\
\hline "NoAnchor-T4( $\backslash=) "$ & $\mathrm{~T} 4(\backslash=)$ & "NoAnchor-T4( $\backslash=) "$ & $\mathrm{~T} 4(\backslash=)$ & & \\
\hline 0.026 & 0.003 & -0.016 & -0.003 & 0.026 & 0.0005 \\
\hline 0.051 & 0.009 & -0.001 & -0.009 & 0.029 & 0.029 \\
\hline 0.115 & 0.008 & 0.106 & -0.008 & 0.051 & -0.003 \\
\hline 0.057 & 0.002 & -0.053 & 0.002 & 0.032 & 0.031 \\
\hline 0.809 & 0.086 & 0.809 & -0.086 & 0.046 & 0.045 \\
\hline 0.923 & 0.056 & 0.820 & -0.056 & 0.042 & 0.032 \\
\hline
\end{tabular}

Table 2: RAD and RD, Treatments: "NoAnchor-T4 $(\backslash=)$ ", "Anchor-T4 $(\backslash=)$ " and KHS . 


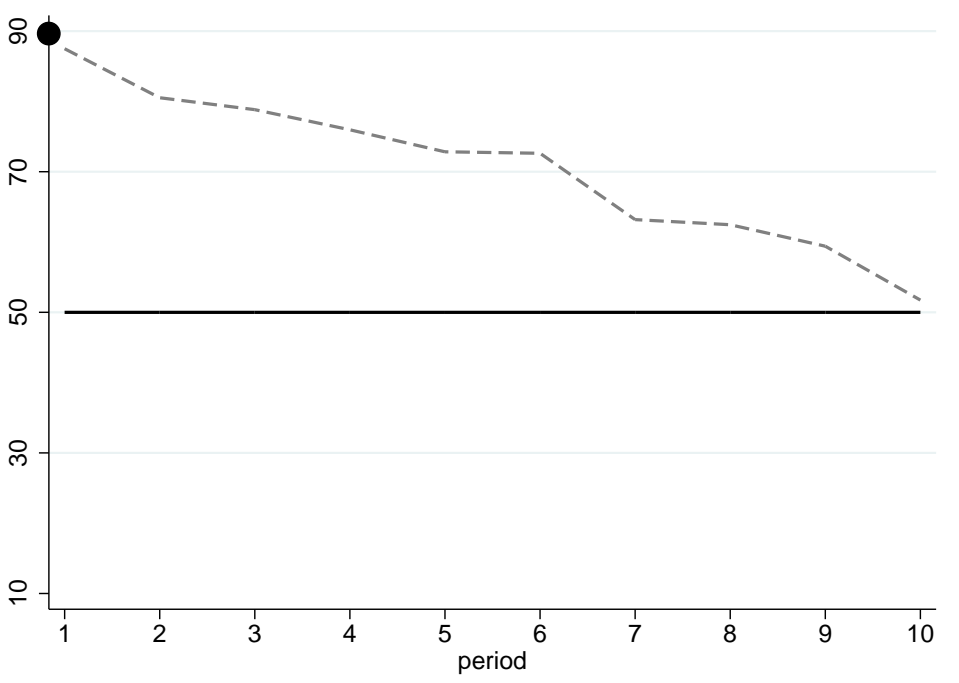

(a) Average Session Prices, "Anchor90-T4 $(\backslash=)$ ".

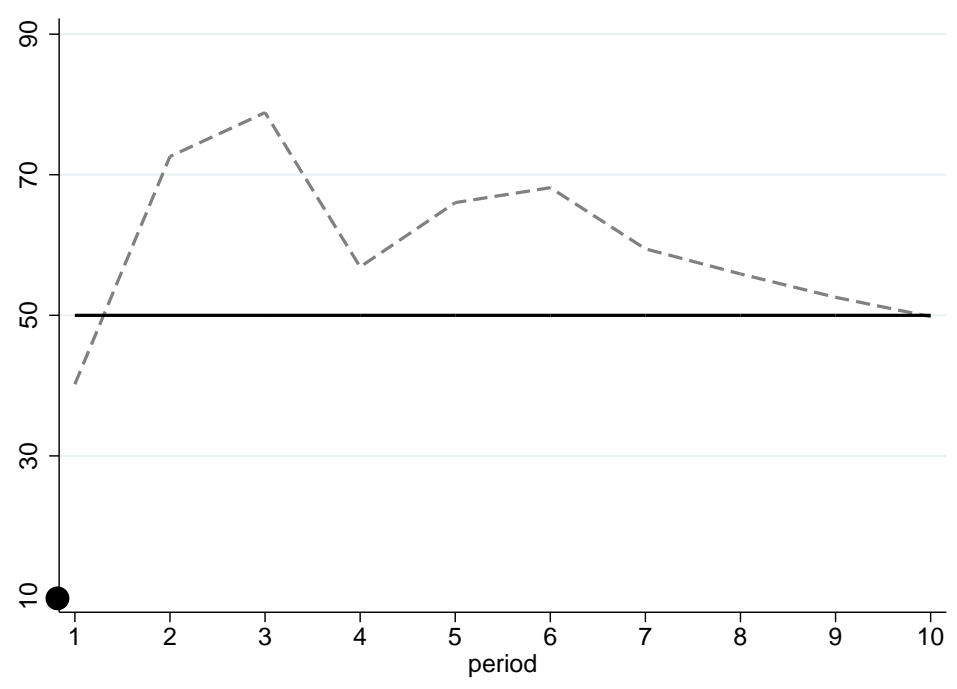

(b) Average Session Prices, "Anchor10-T4 $(\backslash=)$ ".

Figure 2: Dashed Lines: Average Session Prices. Solid Line: FV. Filled Circle: Induced Anchor

treatments differ only in terms of the properties of the graphical interface in the first period. We do not observe significantly less over-valuation (RD). ${ }^{15}$ However, the average RD for our "Anchor"treatment is 0.022 , whereas the average RD for our "NoAnchor" treatment is 0.278 and therefore substantially higher.

Our insights from treatments "Anchor-T4 $(\backslash=)$ " and "NoAnchor-T4( $\backslash=)$ " suggest that subjects are sensitive towards anchors, induced in the first period in experimental asset markets. To test whether subjects are also sensitive towards anchoring if anchors are set at normatively irrelevant numbers, we induced anchors at values of 10 and 90 as described above. We hypothesize that mis-pricing (RAD) is equally severe in both treatments and that over-valuation (RD) is more severe in the "Anchor90-T4( $\backslash=)$ " treatment.

This is indeed what we observe. Table 3 shows session-specific RAD and RD measures for treatments "Anchor90-T4( $=)$ " and "Anchor10-T4( $\backslash=)$ ". A Mann-Whitney test suggests that the RAD-measures do not differ significantly across the two treatments (p-value $=0.151$ ) and that the RD measures in the "Anchor90-T4( $=$ )"-sessions are significantly higher than the RD measures in the "Anchor10-T4 $(\backslash=)$ "-sessions ( -value $=0.095)$.

We observe other interesting patterns. Every single first-period average session price in the "Anchor90-T4( $\backslash=)$ "-treatment exceeds the asset's FV. The average first period price is 87.4 and hence very close to the anchor of 90 . Moreover, four out of the five sessions in the "Anchor90$\mathrm{T} 4(\backslash=)$ "-treatment initiate at high price levels and converge relatively slowly towards the FV,

paths across treatments are particularly pronounced in the first periods. Focusing on periods 1-6 only the p-value is 0.0411 .

${ }^{15}$ Mann-Whitney test $p-$ value $=0.937$. 
suggesting that the anchor is fairly persistent. The fifth session initiates at a value which exceeds the anchor and shows a pronounced bubble. We depict the average session prices for the "Anchor90-T4 $(\backslash=)$ ")-treatment in Figure 2a. Session-specific prices are illustrated in Figures 8a $8 b$.

A different pattern emerges in the "Anchor10-T4 $(\backslash=)$ "-treatment. Four out of five first-period prices in the "Anchor10-T4 $(\backslash=)$ "-sessions initiate below the FV, ranging from 24.56 to 43.7. Most interestingly, each of these four sessions over-shoot and increase beyond the asset's FV at some point in time and converge and/or "crash" back towards the FV towards the end of the session. We depict the average session prices for the "Anchor10-T4( $\backslash=)$ "-treatment in Figure 2b. Sessionspecific prices are illustrated in Figures 8c-8d.

Overall, we conclude that seemingly innocuous visual stimuli can have important effects on price dynamics in experimental asset markets. In the following section we rationalize our findings in the model of Duffy and Ünver [2006] and show that anchoring behavior is an important driver for the observed differences.

\begin{tabular}{|c|c|c|c|}
\hline \multicolumn{2}{|c|}{ RAD } & \multicolumn{2}{|c|}{ RD } \\
\hline "Anchor90-T4( $\backslash=) "$ & "Anchor10-T4 $(\backslash=) "$ & "Anchor90-T4( $(=) "$ & "Anchor10-T4( $\backslash=) "$ \\
\hline 1.456 & 1.078 & 1.456 & 1.039 \\
\hline 0.142 & 0.135 & 0.142 & -0.004 \\
\hline 0.126 & 0.095 & 0.087 & -0.087 \\
\hline 0.183 & 0.047 & 0.183 & 0.047 \\
\hline 0.184 & 0.032 & 0.184 & 0.009 \\
\hline
\end{tabular}

Table 3: RAD and RD for "Anchor90-T4( $=)$ " and "Anchor10-T4( $=)$ "

\section{Exploring Mechanisms: Anchoring}

Duffy and Ünver [2006] (DÜ) provide a model for experimental asset markets, which incorporates anchoring behavior. DÜ model the bid and ask structure of agents in double auctions directly, with a particular focus on experimental setups. Since subjects in experimental asset markets are usually not professional stock traders, they present a parsimonious agent-based model, which captures important behavioral biases commonly observed in laboratory environments.

Although their model might not be compelling enough to capture the behavior of traders in real-world financial markets, it generates very good fits and accurate predictions for experimental asset markets, indicating that standard subject pools stick to "rule-of-thumb" trading in complex asset-market environments. Agent-based models have proven to be powerful tools in explaining phenomena observed in laboratory- and sometimes even in field environments since Gode and 
Sunder [1993]. ${ }^{16}$

To keep the main body of the paper brief, we summarize the four parameters in the model $(\alpha, \kappa, \phi, S)$ and provide a full description of the model in Appendix B.

$\alpha \in[0,1]$ is an anchoring parameter. It captures how intensely agents anchor both their bids and asks towards previous trading prices. In period one, agents anchor their bids and asks to $p_{0}$, which DÜ suggest to be zero. Agents in DÜ also anchor their bids and asks towards a uniformly distributed noise term, $\epsilon_{t} \sim U\left[0, \kappa F V_{t}\right]$, with weight $1-\alpha$. Note that the noise term has mean $\kappa F V_{t} / 2$ and is therefore centered around a multiple of the fundamental value in period $t$. The parameter $\kappa$ measures two things. It measures the multiple of the fundamental value to which subjects anchor their bids and asks and it also measures the noise in bids and asks themselves. One can interpret $\kappa$ as a measure of confusion, reflected in noisy trading behavior.

The parameter $\phi$ captures the notion of foresight. The greater is $\phi$, the quicker the agents switch from buying to selling the asset, taking the finite horizon of the experiment into account. If $\phi$ equals zero, they unsystematically buy or sell in every period with probability $1 / 2$. The parameter $S$ keeps track of the trading volume, measuring the number of offers an agent makes in every trading period.

The model provides the basis to simulate average prices and quantities for every period $t$, which will be denoted as $\bar{p}_{t}(\alpha, \kappa, \phi, S)$ and $\bar{q}_{t}(\alpha, \kappa, \phi, S)$.

DÜ suggest estimating the parameters of the model by minimizing the sum of the squared deviations (SSD) between volume-weighted average experimental prices and quantities, $\left(\bar{p}_{t}, \bar{q}_{t}\right)$, and simulated prices and quantities stemming from the model, normalized by the fundamental value in period one $\left(F V_{1}\right)$ and the total supply of units/shares (TSU):

$$
S S D(\alpha, \kappa, \phi, S)=\sum_{t=1}^{T}\left(\frac{\bar{p}_{t}(\alpha, \kappa, \phi, S)-\bar{p}_{t}}{F V_{1}}\right)^{2}+\sum_{t=1}^{T}\left(\frac{\bar{q}_{t}(\alpha, \kappa, \phi, S)-\bar{q}_{t}}{T S U}\right)^{2}
$$

Duffy and Ünver [2006] use the SSW data and estimate that $\kappa^{*}=4.0846, \alpha^{*}=0.848, \phi^{*}=$ 0.01674 and $S^{*}=5$. Before we proceed using these parameters in the model of DÜ, to understand the underlying mechanism behind the treatment effects in our data, we have to perform several adjustments.

Adjustments. We start by adapting the model environment of DÜ so that our agents can submit bids and asks for multiple units in every period. Details on this procedure are provided in Appendix B. We do this for two reasons. First, in our experiments subjects could submit bids and asks for multiple units, rather than for one unit at a time as in SSW. Second, it allows us to disregard the parameter that keeps track of trading volume $(S)$ for the remainder of the paper and

\footnotetext{
${ }^{16}$ For a review of the links between agent-based models and human subject experiments, see Axelrod and Tesfatsion [2006], Duffy [2006] or Hommes [2006]. Cason [1992] for example uses an agent based model to explain high efficiency levels in experimental call markets.
} 
to focus our analysis on price- rather than volume dynamics. The adjustment on trading volume has little to no effect on trading prices in the DÜ model but facilitates our analysis. We show in Appendix B that we can replicate the simulation results provided in Duffy and Ünver [2006] under our adjusted version of the model.

Lastly, we also have to take into account that in our experiments subjects traded for 10 and not 15 periods as in SSW. For that purpose we adjust the parameter $\phi$ which, as mentioned above, measures the speed at which traders switch from buying to selling behavior. In the DÜ model $\phi=0.01674$ implies that around a quarter of the traders purchase assets in the last period, if the trading horizon is 15 periods. ${ }^{17}$ To obtain a similar fraction of buyers in the last period in our 10 period framework we set $\phi^{*}=0.025\left(\approx 0.01674 \frac{15}{10}\right)$.

\subsection{Baseline-Analysis}

We next simulate the resulting prices in the adapted DÜ model and compare the resulting price dynamics to the ones which emerge in our "NoAnchor-T4 $(\backslash=)$ " treatment. This will allow an assessment of the behavioral changes induced by our design, if compared to the standard SSW framework. Comparing the parameters estimated from the SSW data to the ones which fit prices under treatment "NoAnchor-T4 $(\backslash=)$ " seems most appropriate since no anchors were induced in either setup.

The simulated prices are shown in Figure 3a. The blue line is the resulting price path if $\left(\kappa^{*}, \alpha^{*}, \phi^{*}\right)=(4.0846,0.848,0.025)$. For the sake of completeness we also provide simulated prices under the original DÜ parameters, ${ }^{18}$ to show that our marginal adjustment in our foresight parameter has little effect on the resulting dynamics except for the last few periods (dashed line). Clearly, both price paths do not fit our data generating process. Furthermore, it is also apparent that a univariate change in $\phi$ does generally not result in price patterns which fit our data.

We next investigate the parametric changes which would be necessary to generate price paths fitting our data more closely. We focus on changes in $\kappa$ and $\alpha$, aiming to disentangle confusion from anchoring. We keep our foresight parameter, $\phi$, fixed at 0.025 for the remainder of the analysis. A decrease in $\kappa$ results, ceteris paribus, in lower prices, fitting our data even worse than the ones we have used above. An increase in $\kappa$, consistent with an increase in noise or confusion, leads to an upward shift of prices, without affecting the underlying dynamics. For illustrative purposes we simulate prices in our DÜ model with $\phi^{*}=0.025$ and $\kappa=8$, while leaving $\alpha^{*}=0.848$. The resulting prices are shown in Figure 3 a (red line). We again observe that the simulated prices do not fit our data generating process.

Next, we change our anchoring parameter $\alpha$. As a first step, we simply halve the parameter,

\footnotetext{
${ }^{17}$ The purchase probability for agents is $\pi_{t}=\max \{0.5-\phi(t-1), 0\}$. Specifically, $26.6 \%$ of traders want to purchase assets in the last period. Our results do not change qualitatively, independently of multiplying $\phi$ with $\frac{15}{10}$ or $\frac{14}{9}$.

${ }^{18}$ More specifically: $\left(\kappa^{*}, \alpha^{*}, \phi^{*}\right)=(4.0846,0.848,0.01674)$
} 


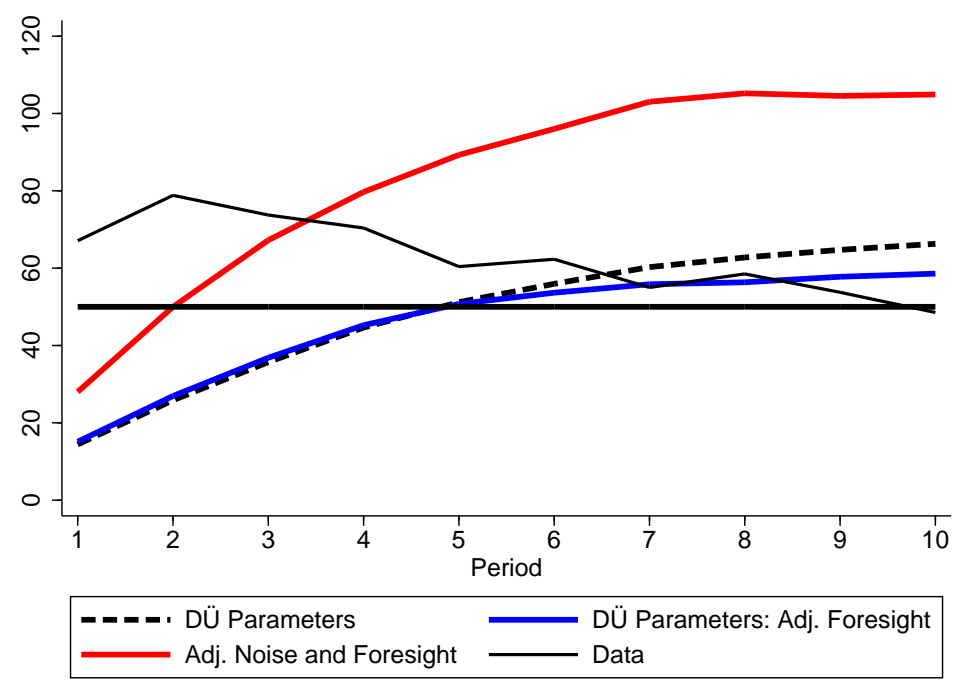

(a)

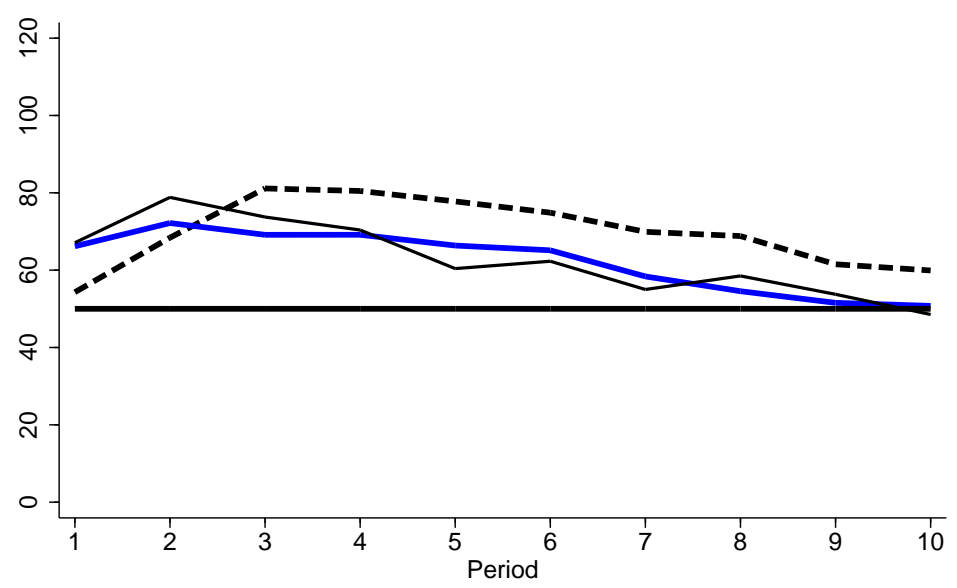

DÜ Parameters: Adjust Anchoring DÜ Parameters: Adjust Noise and Anchoring

(b)

Figure 3: Simulated Average Prices and Actual Average Prices. Panel (a): Horizontal line: FV. Dashed line: Simulated average prices in the DÜ model with $\left(\kappa^{*}, \alpha^{*}, \phi^{*}\right)=(4.0846,0.848,0.01674)$. Blue line: Simulated average prices in the DÜ model with $\left(\kappa^{*}, \alpha^{*}, \phi^{*}\right)=$ $(4.0846,0.848,0.025)$. Red line: Simulated average prices in the DÜ model with $\left(\kappa^{*}, \alpha^{*}, \phi^{*}\right)=(8,0.848,0.025)$. Black line: Average prices from "NoAnchor-T4 $(\backslash=)$ " treatment. Panel (b): Horizontal line: FV. Dashed line: Simulated average prices in the DÜ model with $\left(\kappa^{*}, \alpha^{*}, \phi^{*}\right)=$ $(4.0846,0.424,0.025)$. Blue line: Simulated average prices in the DÜ model with $\left(\kappa^{*}, \alpha^{*}, \phi^{*}\right)=(3.501,0.1675,0.025)($ optimal fit). Black line: Average prices from "NoAnchor-T4( $\backslash=)$ " treatment.

while keeping $\phi^{*}=0.025$ and $\kappa^{*}=4.0846$. This change in parameters can be interpreted as more significant anchoring towards a multiple of the fundamental value. The resulting price dynamics are shown in Figure 3b (dashed line). We observe that the resulting price dynamics still differ quantitatively but capture various stylized features of our average data: Prices initiate above the FV, increase for the first few periods and then start to decrease. ${ }^{19}$

Our comparative statics exercise suggests that, if we start out with the behavioral parameters estimated under the SSW design, we would require a significant increase of anchoring towards a multiple of the FV to fit our data more closely. Indeed, in the last step of our univariate analysis we also minimize (1) ignoring trading volumes and using a grid point minimization procedure as suggested in Duffy and Ünver [2006]. In this procedure we fix $\phi^{*}=0.025$ and $\kappa^{*}=4.0846$ and estimate $\alpha^{*}$. The optimal $\alpha^{*}$ takes a value of 0.162 , further suggesting that the design of "NoAnchor-T4 $(\backslash=)$ " favors anchoring towards a multiple of the FV.

Our analysis from above suggests that a univariate change in our "confusion" parameter, $\kappa$, does not generate price paths which capture basic features of our data. Hence, in contrast to the SSW design, the design of treatment "NoAnchor-T4 $(\backslash=)$ " does not necessarily reduce confusion as parametrized via $\kappa$.

In a last step, we estimate the parameter values of $\kappa$ and $\alpha$, which jointly minimize (1), while ignoring trading volume and without changing our foresight parameter. In other words, we

\footnotetext{
${ }^{19}$ Note that the average initial price in DÜ equals $(1-\alpha) \kappa F V / 2=58.8182$.
} 
fix $\phi^{*}=0.025$ in our minimization procedure. The resulting parameter estimates are given by $\kappa^{*}=3.501$ and $\alpha^{*}=0.1675$. We observe that, relative to the SSW design, the estimated confusion parameter decreases marginally, whereas anchoring towards a multiple of the FV increases almost by a factor of 5.5. The latter finding is at least indicative that the design under our "NoAnchor$\mathrm{T} 4(\backslash=)$ " treatment could trade of confusion, potentially emerging under the standard SSW design, with anchoring towards a multiple of the FV.

In summary, even without inducing an anchor in the first period via visual stimuli, the design under our "NoAnchor-T4 $(\backslash=)$ " treatment seems to favor anchoring towards a multiple of the FV. The simulated prices for $\left(\kappa^{*}, \alpha^{*}, \phi^{*}\right)=(3.501,0.1675,0.025)$, i.e. our optimal fits, are shown in Figure $3 \mathrm{~b}$ (blue line). In the following we will use this final set of parameters to reconcile various features of our data, which emerge under our treatment variations. ${ }^{20}$

\subsection{Asymmetric Price Dynamics}

One interesting feature of the price data from our experiments is the asymmetry in the price paths followed by low (10) and high (90) initial anchors. Low anchors induce increasing prices, peaking most of the time above the FV and crashing/converging to the FV thereafter.

We show next that we can reconcile the observed asymmetries in the model of DÜ, at least qualitatively. First, we fix our final set of parameters, estimated under our "NoAnchor-T4 $(\backslash=)$ " treatment, and simply vary the initial (non-trading) price $p_{0}$. Note that $p_{0}$ affects initial transaction prices in the model, but is not a transaction price itself. It is the price towards which agents, in the model, anchor their bids and asks in the first period. Hence, $p_{0}$ can be interpreted as the natural theoretical analogue to the visual stimulus induced in our within period price charts in the first period. We therefore set $p_{0}$ to 90,50 and 10 to capture our laboratory treatment variations in the model, assuming unchanged behavioral parameters. The resulting price paths are shown in Figure $4 \mathrm{a}$.

Similar to our experimental data, we observe that initial simulated prices are increasing in $p_{0}$. Simulated prices are initially lowest if $p_{0}$ is set to 10 and are highest if we set $p_{0}$ to 90 . Price paths in which $p_{0}$ is set at 50 initiate in between. Moreover, we observe that prices are decreasing towards the $\mathrm{FV}$ if we set a high initial anchor in our model $\left(p_{0}=90\right)$. We also observe that prices are initially increasing over the first few periods and then start to decrease if we set the $p_{0}$ to 10 . Hence, simulated prices are more hump shaped under an initial anchor of 10 than under an initial anchor of 90 , consistent with our data.

The mechanism behind this asymmetry in the model is fairly straightforward. The limiting price in the model is itself a decreasing function of time whenever $\phi>0$. By initiating prices closer towards this limit, they descend over the entire trading horizon. By initiating prices below

\footnotetext{
${ }^{20}$ Figure $6 \mathrm{~b}$ in Appendix B shows the surface of the our objective function in (1), disregarding volume dynamics, around the minimum.
} 


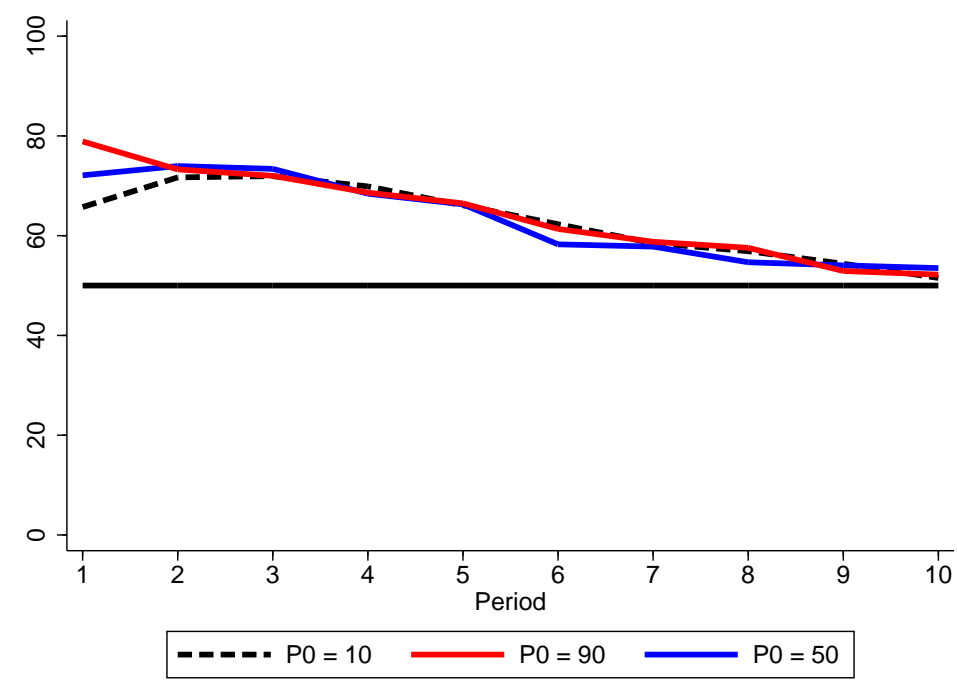

(a)

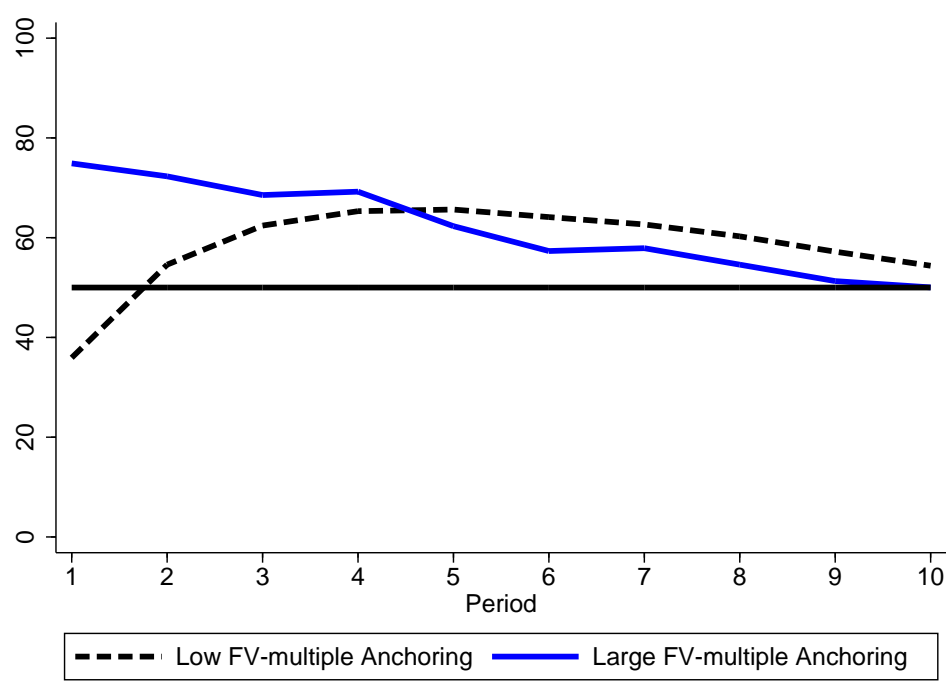

(b)

Figure 4: Simulated Average Prices and treatment variations. Panel (a): Horizontal line: FV. For all simulations we used $\left(\kappa^{*}, \alpha^{*}, \phi^{*}\right)=$ $(3.501,0.1675,0.025)$. Dashed line: Simulated average prices in the DÜ model with $p_{0}=10$. Blue line: Simulated average prices in the DÜ model with $p_{0}=50(\mathrm{FV})$. Red line: Simulated average prices in the DÜ model with $p_{0}=90$. Panel (b): Horizontal line: FV. Dashed line: Simulated average prices in the DÜ model with low anchoring towards a multiple of the FV $\left(\kappa^{*}, \alpha^{*}, \phi^{*}\right)=(3.501,0.55,0.025)$. Blue line: Simulated average prices in the DÜ model with large FV-anchoring $\left(\kappa^{*}, \alpha^{*}, \phi^{*}\right)=(3.501,0.01,0.025)$.

the long-run price, we first observe that transaction prices first increase towards the limit from below until they converge and also start to decrease along the long run limiting price path. Put differently, we can reconcile the asymmetries in the price paths observed in our data in the model of DÜ, at least qualitatively, simply by varying initial anchors. However, there are two important features of our data that we cannot capture in the model by varying only $p_{0}$.

The first feature, which the model does not generate by varying only $p_{0}$, is that prices tend to initiate below the FV in our low anchor treatment. This suggests that our treatment variation, which operates in the model through changes in $p_{0}$, also affects some of the underlying behavioral parameters. We will next show that the change in behavior is primarily driven by a change in our anchoring parameter, $\alpha$.

Specifically, we first change the anchoring parameter, while keeping all other parameters fixed as estimated under our "NoAnchor-T4 $(\backslash=)$ " treatment. We also keep the initial price, $p_{0}$, in all simulations fixed at a value of zero, just like in our initial estimation. ${ }^{21}$ Under the latter assumption, a decrease in $\alpha$ is equivalent to anchoring bids and asks stronger towards a multiple of the underlying fundamental value. As a matter of fact, a change in $\alpha$ has two effects in the model. First, it changes the average initial price $\left(=(1-\alpha) \frac{\kappa F V}{2}\right)$ and second, it also changes the price dynamics. The resulting price paths are shown in Figure $4 \mathrm{~b}$.

We observe that an increase in anchoring towards a multiple of the FV results in higher initial

\footnotetext{
${ }^{21}$ However, our results do not change qualitatively if we would also adjust $p_{0}$ based on our treatment variations. I.e. if we would set $p_{0}$ equal to 10 or 90 , depending on the change in $\alpha$.
} 
prices followed by a decreasing price path. A decrease in anchoring towards the same multiple, on the other hand, generates hump shaped price paths which initiate below the FV. In other words, by varying the prime anchoring parameter in the model we can generate asymmetric price paths, with the additional feature that prices initiate below the FV, consistent with our data. Our model suggests that low initial anchors - set below the FV-favor past price anchoring, resulting in bubble-crash patterns, similar to the ones we observe under our "NoAnchor-T4 $(\backslash=)$ " treatment, although more pronounced.

Importantly, no other univariate change in parameters in the model can generate these differences in dynamics or captures the observed features of our data appropriately. A change in $\kappa$ results in level shifts of prices, without affecting the dynamics. A change in $\phi$ has no effect on initial prices. Put differently, using our "NoAnchor-T4 $(\backslash=)$ " treatment parameters as benchmark, we cannot reconcile the observed price asymmetries in our data solely by varying $\kappa$ or $\phi$. Only changes in $p_{0}$ or $\alpha$ generate patterns qualitatively consistent with our data. The variable $p_{0}$ is the theoretical analogue to our treatment variation on initial prices in the experiments. The variable $\alpha$ captures anchoring behavior. In summary, these findings therefore indicate that the mechanism behind our observed treatment effects is a change in anchored bidding behavior, induced via visual stimuli in the first period.

However, there is one feature in our data, which cannot be reconciled easily in the model of DÜ. In the model, initiating prices in the FV does not result in prices which remain at the fundamental value. They accurately initiate in between initial prices under low (10) and high (90) initial anchors but do not remain anchored at the FV. In the model, this feature can only be generated by changing several behavioral parameters simultaneously. If $p_{0}=F V$ subsequent prices only remain close to the $\mathrm{FV}$ by setting $\kappa \approx 2$ and $\phi \approx 0 .{ }^{22}$ Hence, we would need various simultaneous changes in parameters to capture the fact that prices stay close to the FV, whenever initiated there. More specifically, we would require a reduction in the noise amplitude, coupled with random sales and purchase decisions.

We conclude that we can reconcile most of the features from our experiments in the model of DÜ. In most of the cases, whenever we require changes in behavioral parameters to reconcile some of our treatment effects, these changes are primarily affecting our prime anchoring parameter. This evidence suggests that our treatment effects primarily operate, as proposed, through anchored bidding behavior.

Momentum approach. One potential criticism against the approach taken above is that price asymmetries emerge rather mechanically in the DÜ model. We therefore present an alternative, although related, model in Appendix D which is based on Caginalp et al. [2000] (CPS hereafter).

\footnotetext{
${ }^{22}$ Note that if $\phi=0$, the long run price is $\kappa F V / 2$, which is equal to $F V$ iff $\kappa=2$. If also $p_{0}=F V$, initial bids and asks are on average equal to the FV and remain at this value for any $\alpha$. We refer to the Appendix for details on the model.
} 
The model captures the idea that initial prices affect subsequent equilibrium price dynamics via momentum trading. The reduced model formalizes the general notion that an initial undervaluation of assets generates purchase decisions of some traders, leading to a boom of prices. In the model, the upward trend of prices can lead to an overshooting beyond the FV, via momentum purchasing behavior. One can interpret the latter behavior as being generated by trend chasing or momentum-based trader types as in DeLong et al. [1990]. The resulting boom turns into a crash if prices are sufficiently high, and the cash levels of purchasing traders are sufficiently low. This interplay between low cash levels and overvalued assets leads to selling behavior in the model, inducing a crash of prices. In Appendix D we show that this model can also generate asymmetric price paths, similar to the ones we observe in our data, after incorporating our anchoring insights. In contrast to the DÜ model, the CPS model can also generate the observation that prices stay at the $\mathrm{FV}$ whenever initiated there, without varying additional model parameters. However, in contrast to the DÜ model, we require a simultaneous change in initial prices and parameters to generate asymmetric price dynamics in the CPS model.

\subsection{Discussion}

In summary, we conclude from our experiments that visual stimuli, provided in within-period price charts in the first period only, have significant effects on price dynamics. Our results do not only suggest that subjects are sensitive towards these stimuli, but that they can be fairly persistent, generating anchored trading prices. Anchors set at levels which exceed the FV, lead to significantly more over-valuation than anchors set at levels which are below the FV. More specifically, initial over- and undervaluation, generated via anchoring, lead to asymmetric price responses. Low anchors generate prices which initiate below the FV, overshoot at some point and converge/crash to the FV thereafter. High anchors induce prices which slowly converge to the FV from above. Anchoring prices towards the fundamental value in the first period, via visual stimuli, mitigates bubbles in our experiments. Lastly, if no anchors are induced in the first period, prices tend to show standard bubble-crash patterns usually observed in laboratory asset markets, even if the FV is constant.

Our estimation results from the DÜ model confirm our initial assessment that the mechanism through which visual stimuli affect prices is indeed via anchored bidding behavior. The effects are particularly strong for treatments in which stimuli were induced above or below the FV. The DÜ model suggests that the bubble mitigation effect, observed whenever we induced an anchor at the FV, is driven by a combination of behavioral changes such as anchoring, a reduction in noisy bidding behavior, coupled with random purchase and sales decisions. In other words, setting an anchor at the FV has complex effects on various aspects of individual trading behavior and hence prices.

Initial price anchoring, as observed in this study, is a phenomenon which is also important 
to understand stock-price dynamics and not just experimental environments. For instance, news about the underlying fundamental value of a stock can move stock prices (Caginalp et al. [2008]), potentially resulting in anchored bidding behavior and thereby affecting subsequent prices.

Initial daily trading prices, established by market-makers during pre-openings, can have similar effects on bidding behavior. More specifically, our results suggest that initial over- or underpricing can generate asymmetric and non-trivial price responses.

Our insights also suggest that IPO-prices may potentially affect subsequent prices of the underlying stock via anchored bidding behavior. Initial IPO-underpricing may result into price-rallies whereas initial over-pricing could persist for extended periods of time.

Lastly, graphical representations of trading prices are omnipresent in the banking industry. Our stylized laboratory framework suggests that the particular features of these visualizations can have nontrivial effects on individual trading behavior and hence prices.

\section{Conclusion}

We show that setting an anchor at the FV in price charts, in the first period only, can be sufficient to eliminate or to significantly mitigate bubbles in experimental asset markets. Our results further indicate that persistent mis-pricing of assets in laboratory environments is primarily sparked by an initial deviation of asset prices from the homogeneous beliefs, rational expectations equilibrium. Initial over- and undervaluation, generated via anchoring, lead to asymmetric price responses. Low anchors generate prices which initiate below the FV, overshoot at some point and converge/crash to the FV thereafter. High anchors induce prices which slowly converge to the FV from above. We rationalize our findings in the models of Duffy and Ünver [2006] and Caginalp et al. [2000]. We argue that our results can be related to IPO's and the relationship between opening prices and intra-day price dynamics on stock exchanges.

\section{References}

Y. Amihud and H. Mendelson. Trading mechanisms and stock returns: An empirical investigation. Journal of Finance, 42:533-553, 1987.

D. Ariely, G. Loewenstein, and D. Prelec. Coherent arbitrariness: Stable demand curves without stable preferences. Quarterly Journal of Economics, 118(1):73-106, 2003.

R. Axelrod and L. Tesfatsion. A guide for newcomers to agent-based modeling in the social sciences, in: L. Tesfatsion and K.L. Judd, eds., Handbook of Computational Economics Vol. 2 Handbooks in Economics Series. Elsevier/North-Holland (Handbooks in Economics Series), 2006. 
B. Biais, C. Bisière, and S. Pouget. Equilibrium discovery and preopening mechanisms in an experimental market. Management Science, 60(3):753-769, 2013.

Bruno Biais, Pierre Hillion, and Chester Spatt. Price discovery and learning during the preopening period in the paris bourse. Journal of Political Economy, 107(6):1218-1248, 1999.

M.V. Boening, S. LaMaster, and A. Williams. Price bubbles and crashes in experimental call markets. Economics Letters, 41:179-185, 1993.

R.H. Byrd, M. C. Hribar, and J. Nocedal. An interior point algorithm for large-scale nonlinear programming. SIAM Journal on Optimization, 9:877-900, 1999.

R.H. Byrd, J. C. Gilbert, and J. Nocedal. A trust region method based on interior point techniques for nonlinear programming. Mathematical Programming, 89:149-185, 2000.

G. Caginalp and V. Ilieva. The dynamics of trader motivations in asset bubbles. Journal of Economic Behavior and Organization, 66(3-4):641-656, 2005.

G. Caginalp, D. Porter, and V. L. Smith. Initial cash to asset ratio and asset prices. an experimental study. PNAS, 95(2):756-761, 1998.

G. Caginalp, D. Porter, and V. L. Smith. Momentum and overreaction in experimental asset markets. International Journal of Industrial Organization, 18(1):187-204, 2000.

G. Caginalp, L. Hao, D. Porter, and V. L. Smith. Asset market reactions to news: an experimental study. Chapman University, Economic Science Institute Working Papers, 2008.

T. Cason. Call market efficiency with simple adaptive learning. Economics Letters, 40:27-32, 1992.

T. N. Cason and A. S. Samek. Visual representation and observational learning in asset market bubbles. 2014 .

J. B. DeLong, A. Shleifer, L. H. Summers, and R. J. Waldman. Positive feedback investment strategies and destabilizing rational speculation. Journal of Finance, 45:379-395, 1990.

J. Duffy. Agent-Based Models and Human Subject Experiments, in: L. Tesfatsion and K.L. Judd, eds., Handbook of Computational Economics Vol. 2 Handbooks in Economics Series. Elsevier/North-Holland (Handbooks in Economics Series), 2006.

J. Duffy and U. M. Ünver. Asset price bubbles and crashes with near-zero-intelligence traders. Economic Theory, 27:537-563, 2006.

M. Dufwenberg, T. Lindqvist, and E. Moore. Bubbles and experience: An experiment. American Economic Review, 95(5):1731-1737, 2005. 
Ann B. Gillette, Douglas E. Stevens, Susan G. Watts, and Arlington W. Williams. Price and volume reactions to public information releases: An experimental approach incorporating traders' subjective beliefs. Contemporary Accounting Research, 16(3):437-479, 1999.

D. K. Gode and S. Sunder. Allocative efficiency of markets with zero-intelligence traders: Market as a partial substitute for individual rationality. Journal of Political Economy, 101(1):119-137, 1993.

E. Haruvy and C. N. Noussair. The effect of short selling on bubbles and crashes in experimental spot asset markets. Journal of Finance, 61(3):1119-1157, 2006.

E. Haruvy, Y. Lahav, and C. N. Noussair. Traders' expectations in asset markets: Experimental evidence. American Economic Review, 97(5):1901-1920, 2007.

C. Hommes. Heterogeneous Agent Models in Economics and Finance, in: K.L. Judd and L. Tesfatsion, eds., Handbook of Computational Economics Vol. 2: Agent-Based Computational Economics. Elsevier/North-Holland (Handbooks in Economics Series), 2006.

R. N. Hussam, D. Porter, and V. L. Smith. Thar she blows: Can bubbles be rekindled with experienced subjects? American Economic Review, 98(3):924-937, 2008.

D. Kahneman and A. Tversky. Judgement under uncertainty: heuristics and biases. Science, 185: 1124-1131, 1974.

D. Kahneman and A. Tversky. Extensional versus intuitive reasoning: the conjunction fallacy in probability judgement. Psychological Review, 90:293-315, 1983.

R. King, V. L. Smith, A. Williams, and M. v. Boening. Nonlinear Dynamics and Evolutionary Economics. Oxford University Press, 1993.

M. Kirchler, J. Huber, and T. Stöckl. Bubble measures in experimental asset markets. Experimental Economics, 13:284-298, 2010.

M. Kirchler, J. Huber, and T. Stöckl. Thar she bursts: Reducing confusion reduces bubbles. American Economic Review, 102(2):865-883, 2012.

T. Loughrand and J. R. Ritter. Why don't issues get upset about leaving money on the table in ipo's. Review of Financial Studies, 15(2):413-444, 2002.

C. N. Noussair and S. Tucker. Futures markets and bubble formation in experimental asset markets. Pacific Economic Review, 11(2):167-184, 2006.

C. N. Noussair, S. Robin, and Ruffieux B. Price bubbles in laboratory asset markets with constant fundamental value. Experimental Economics, 4:87-105, 2001. 
D. Porter and V. L. Smith. Futures markets and dividend uncertainty in experimental asset markets. Journal of Business, 68(4):509-541, 1995.

D. Porter, G. Caginalp, and L. Hao. Asset market reactions to news: An experimental study. 2010.

O. Powell. Eye tracking in the market, subject focus in experimental bubble markets. 2010.

J. R. Ritter and I. Welch. A review of ipo activity, pricing and allocations. The Journal of Finance, 58(4):1795-1828, 2002.

V. L. Smith, G. L. Suchanek, and A. Williams. Bubbles, crashes, and endogenous expectations in experimental spot asset markets. Econometrica, 56(5):1119-1151, 1988.

V. L. Smith, G. Caginalp, and D. Porter. Overreaction, momentum, liquidity, and price bubbles in laboratory and field asset markets. The Journal of Psychology $\& 3$ Financial Markets, 1:24-48, 2000 .

H. Stoll and R. Whaley. Stock market structure and volatility. Review of Financial Studies, 3: 37-71, 1990.

A. Williams. Price Bubbles in Large Financial Asset Markets? Handbook of Experimental Economics Results, 2008. 


\section{Appendix A (For Online Publication)}

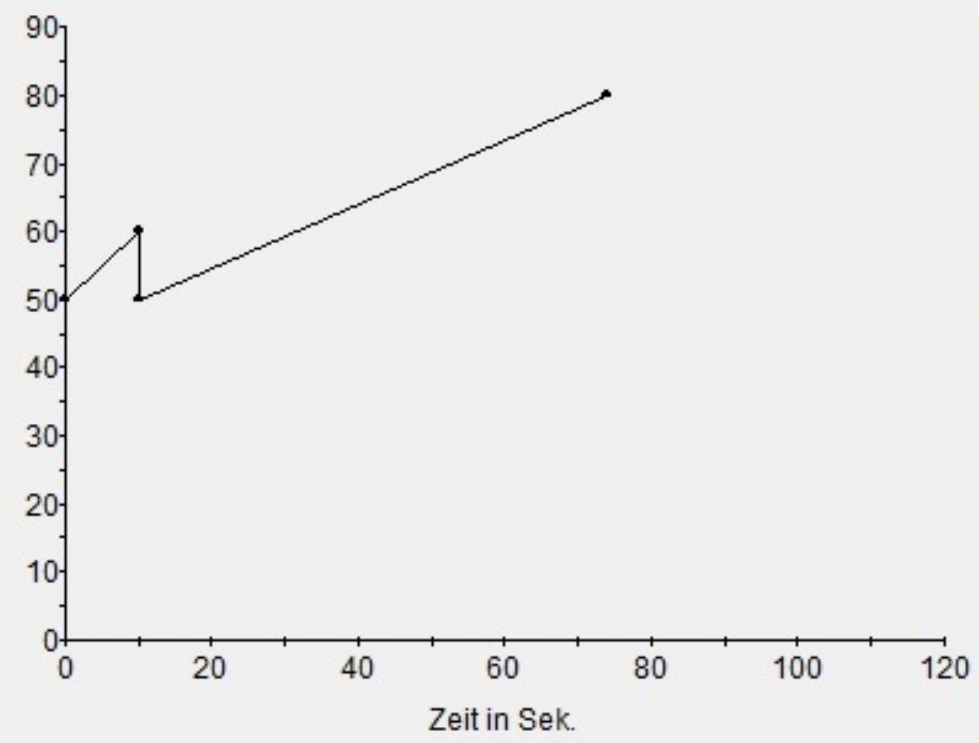

(a) First Price $>F V=50$

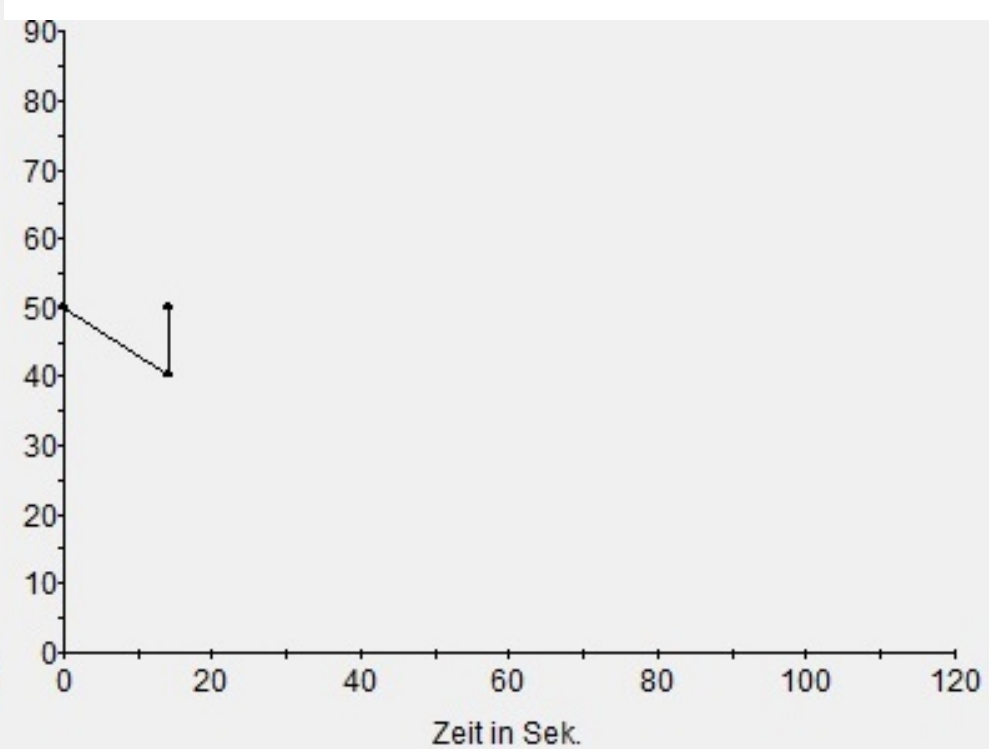

(b) First Price $<F V=50$

Figure 5: Price Initiation and jump-back component, KHS (Screenshots, Experimental software).

\section{Appendix B (For Online Publication)}

In this section, the market environment as well as agents' behavior are described, following Duffy and Ünver [2006].

In the market environment of KHS, $N=10$ agents interact in $T=10$ periods and trade a single financial asset. Initially each agent $i$ is endowed with $x_{0}^{i}$ units of cash and $y_{0}^{i}$ units of the financial asset (for details on the endowment design we refer to KHS).

At the end of every period the asset pays random dividends drawn with equal probability from a commonly known support $\Theta_{D}$. The expected dividend is denoted as $\bar{d}$. The fundamental value of the asset in every period is common knowledge and given by

$$
F V_{t}=\bar{d}(T-t+1)+T V \quad \text { for } \quad t=1, \ldots, T
$$

where TV denotes the terminal value of the asset. Note that the notation is sufficiently flexible to cover the decreasing, as well as the constant FV framework. Under rational expectations and risk neutrality, prices should equal the fundamental value.

In every trading period $t=1, \ldots, T$ traders may either buy or sell units of the financial asset (or remain inactive). During a standard double auction, traders are allowed to submit both bids and asks, potentially for multiple units. To capture this feature in the simulations, Duffy and Ünver 
[2006] subdivide each trading period into $S$ submission rounds.

In each of the $s=1, \ldots, S$ submission rounds a trader is either a seller or a buyer with probability $\pi_{t}=\max \{0.5-\phi(t-1), 0\}$, where $\phi \in[0,0.5 / T)$. Trader $i$ in submission round $s$ and trading period $t$ can submit an ask price, $a_{s, t}^{i}$, for $q_{a, s, t}^{i}$ units of the asset if he is a seller and may submit a bid, $b_{s, t}^{i}$ for $q_{b, s, t}^{i}$ units of the asset, if he is a buyer. Bids can only be submitted if the trader has a sufficient cash balance and asks can only be submitted if the trader has a positive unit-balance. Note that in DÜ, agents can only submit bids and asks for one unit of the asset (as in the standard SSW-design). However, subjects in KHS were allowed to submit bids and asks for several units, which is why we adapted the DÜ environment. The bids in DÜ take the following form:

$$
b_{s, t}^{i}=\min \left\{(1-\alpha) \epsilon_{t}+\alpha p_{t-1}, x_{s, t}^{i}\right\},
$$

where $\alpha \in[0,1], \epsilon_{t} \sim U\left[0, \kappa F V_{t}\right], \kappa \geq 0$ is a parameter, $x_{s, t}^{i}$ denotes the current cash holdings of agent $i$, and $p_{t-1}$ is the market-clearing price in period $t-1 .^{23}$ No bid is submitted by a buyer whenever his cash holdings are zero, i.e., if $x_{s, t}^{i}=0$. The bid quantities are determined as follows:

$$
q_{b, s, t}^{i}=\min \left\{B_{i}, T S U-y_{s, t}^{i}\right\} \quad B_{i} \sim \bar{U}\left[0, \frac{x_{s, t}^{i}}{b_{s, t}^{i}}\right],
$$

where $\bar{U}$ denotes the discrete uniform distribution, TSU is the total stock of units in the market and $y_{s, t}^{i}$ are the units currently held by trader $i$. If the realization of $B_{i}$ equals zero, that trader did not submit a bid.

Similarly, if a trader is selected to be a seller in trading round $t$ and submission period $s$, he submits an ask subject to unit holdings. His ask is of the form

$$
a_{s, t}^{i}=(1-\alpha) \epsilon_{t}+\alpha p_{t-1}
$$

where $\alpha \in(0,1)$ and $\epsilon_{t} \sim U\left[0, \kappa F V_{t}\right]$. No ask is submitted by the seller whenever his unit holdings are zero, i.e., if $y_{s, t}^{i}=0$. The ask quantities are determined as follows:

$$
q_{a, s, t}^{i}=A_{i} \quad A_{i} \sim \bar{U}\left[0, y_{s, t}^{i}\right]
$$

where $\bar{U}$ denotes the discrete uniform distribution and $y_{s, t}^{i}$ are the units currently held by trader $i$. If the realization of $A_{i}$ equals zero, that trader did not submit an ask.

Trading proceeds as follows (we provide a verbal description here, a technical description can be found in Duffy and Ünver [2006] and in the detailed documentation of the Matlab code provided in the supplemental material): In each trading round $s$ of period $t$, traders submit either bids or asks (depending on whether they are buyers or sellers), which are collected in the order book. The highest bid price is the current best bid and the highest ask is the current best ask together with

\footnotetext{
${ }^{23} \mathrm{D} \ddot{\mathrm{U}}$ assume that $p_{0}=0$.
} 
the associated quantities. Orders are ranked conditional on price.

If there is currently a best ask available (see below) and a buyer submits a bid, that exceeds the current best ask (indicating that he is willing to pay more than the current best ask), trade takes place at the current best ask. If the bidding quantities exceed the associated offer-quantities (individual demand would potentially exceed individual supply at the best current ask), the second lowest ask price and the associated quantities become the best current ask offer (the entire order was filled, a new order needs to become the best current ask). Note that the trading quantities in this case are capped at the corresponding ask-quantities, not the demand quantities. ${ }^{24}$ If the bid-quantities do not exceed the offer-quantities, (e.g.: the current best ask involves 12 units and the buyer only wants to purchase 5 units), trade takes place at the current best ask price and the bid-quantities (demand) are filled. The offer quantities are appropriately updated but the current best ask price remains unchanged (in the previous example the ask quantities are reduced to 7). If a buyer submits a bid, which does not exceed the best current ask price but is higher than all other bids in the order book, that buyers bid becomes the best current bid (price and quantity). Otherwise the bid is collected in the order book.

If there is a best current bid available and a seller submits an ask price that is lower than the best current bid price (indicating that he is willing to sell at prices below the current best ask) , trade takes place at the current best bid. If the ask quantities exceed the associated best-bidquantities (individual supply would potentially exceed individual demand at the best current bid), the second highest bid price and the associated quantities become the best current bid offer (the entire order was filled, a new order needs to become the best current bid). Note that the trading quantities in this case are capped at the corresponding bid-quantities, not the supply quantities. ${ }^{25}$ If the ask-quantities do not exceed the best-bid-quantities, (e.g.: the current best bid involves 12 units and the seller only wants to sell 5 units), trade takes place at the current best bid price and the ask-quantities (supply) are delivered. The best-bid-quantities are appropriately updated but the current best bid price remains unchanged (in the previous example the bid quantities are reduced to 7). If a seller submits an ask, which is not below the best current ask price but is lower than all other asks in the order book, that sellers bid becomes the best current ask (price and quantity). Otherwise the ask is collected in the order book.

After every trade, the cash and unit balances are appropriately updated. At the end of the period (after $S$ trading rounds) the cash holdings are also updated. In the standard SSW-design this would imply that:

$$
x_{1, t+1}^{i}=x_{S, t}^{i}+D_{t} y_{S, t}^{i}
$$

where $D_{t}$ is the dividend realization in period $t$. In our environment dividend payments are

\footnotetext{
${ }^{24}$ If there is no second lowest ask price in the order book (no ask available), then the next submitted ask price (which is not lower than the best current bid price), becomes the best current ask price.

${ }^{25}$ If there is no second highest bid price in the order book (no bid available), then the next submitted bid price (which is not higher than the best current ask price), becomes the best current bid price.
} 
not added to the cash holdings.

Figure 6a shows simulated prices under the estimated parameters of DÜ, using the design parameters of SSW. However, we use our adjustment in trading volumes in the model to simulate the prices depicted in the picture. The Figure replicates the corresponding lines in Figure 1 in Duffy and Ünver [2006] to illustrate that our change in volume has little to no effect on the resulting price dynamics.

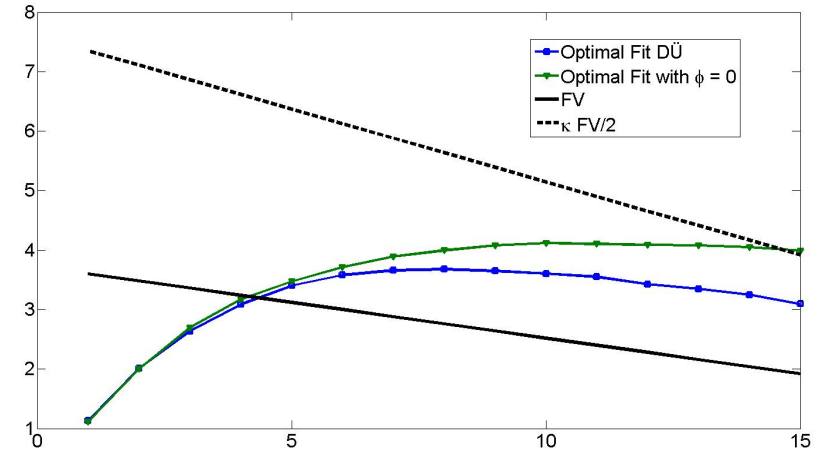

(a) Replicating DÜ. See Figure 1 in Duffy and Ünver [2006].

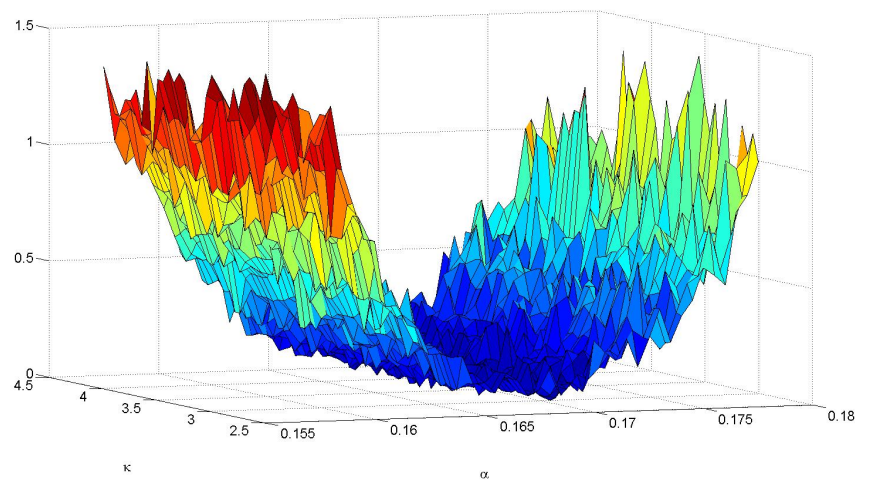

(b) Surface of the objective function in (1) around the minimum, disregarding volume.

Figure 6: Computational aspects. Panel (a) replicates DÜ, using our adjusted volume mechanism. Panel (b) shows a surface plot of our objective function in (1) around the minimum, using our own data and disregarding trading volume. 


\section{Appendix C (For Online Publication)}

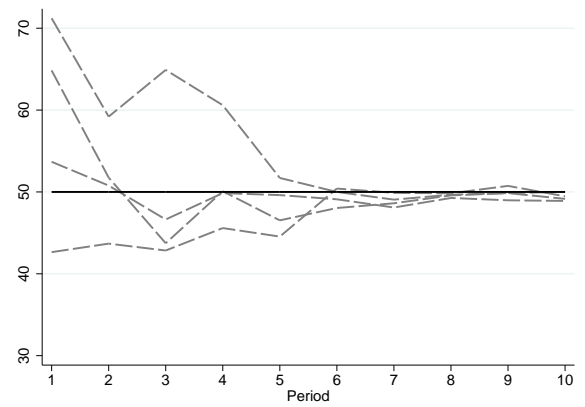

(a) Session Prices $(1-4)$; NoAnchor.

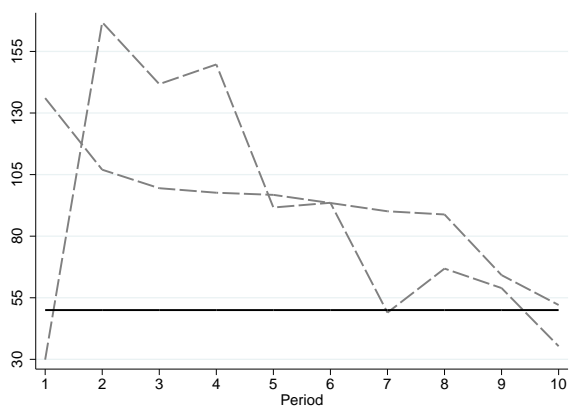

(b) Session Prices (5 - 6); NoAnchor.

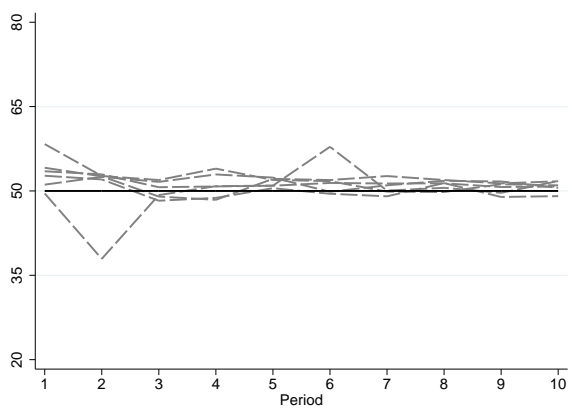

(c) Session Prices (1 - 6); Anchor

Figure 7: Dashed Lines: Session-Prices. Solid Lines: FV (50). Figures 7a - 7b: NoAnchor. Figure 7c: Anchor.

Figures $7 \mathrm{a}-7 \mathrm{~b}$ show the session specific, volume-weighted average transaction prices in the "NoAnchor"-treatment. Figure 7a shows prices for four of our sessions, which also include the sessions with 7 and 9 subjects. Figure $7 \mathrm{~b}$ shows prices of 2 fairly extreme sessions under our "NoAnchor" treatment (Both sessions had 10 subjects). Figure 7c shows the price dynamics for our "Anchor"-treatments. Figures 8a - 8d show the session specific, volume-weighted average transaction prices in the "Anchor90"- and "Anchor10"-treatments. 


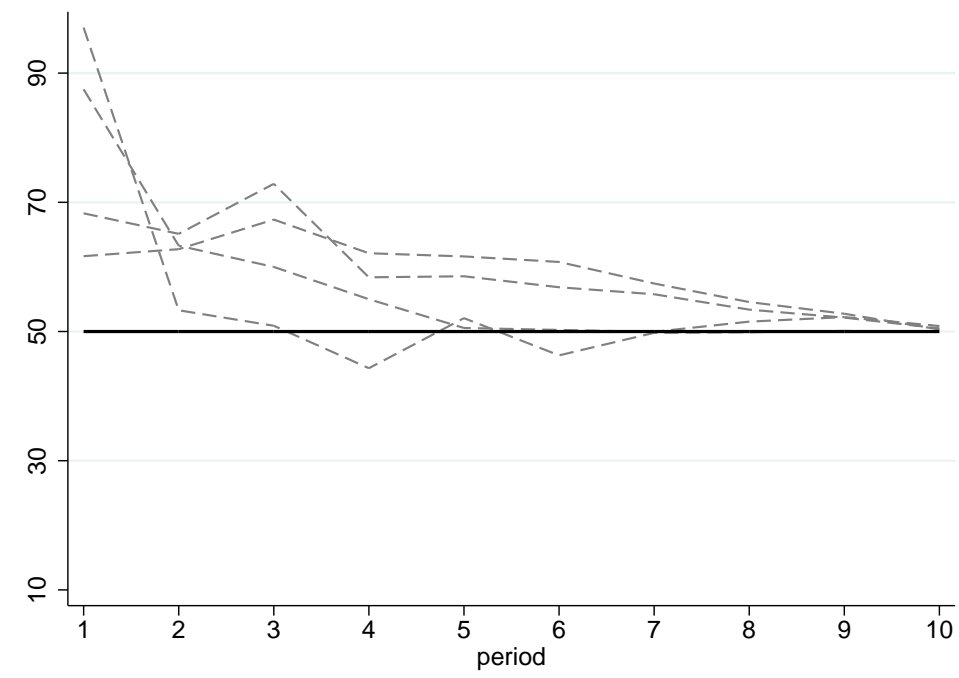

(a) 4 regular sessions: Anchor90

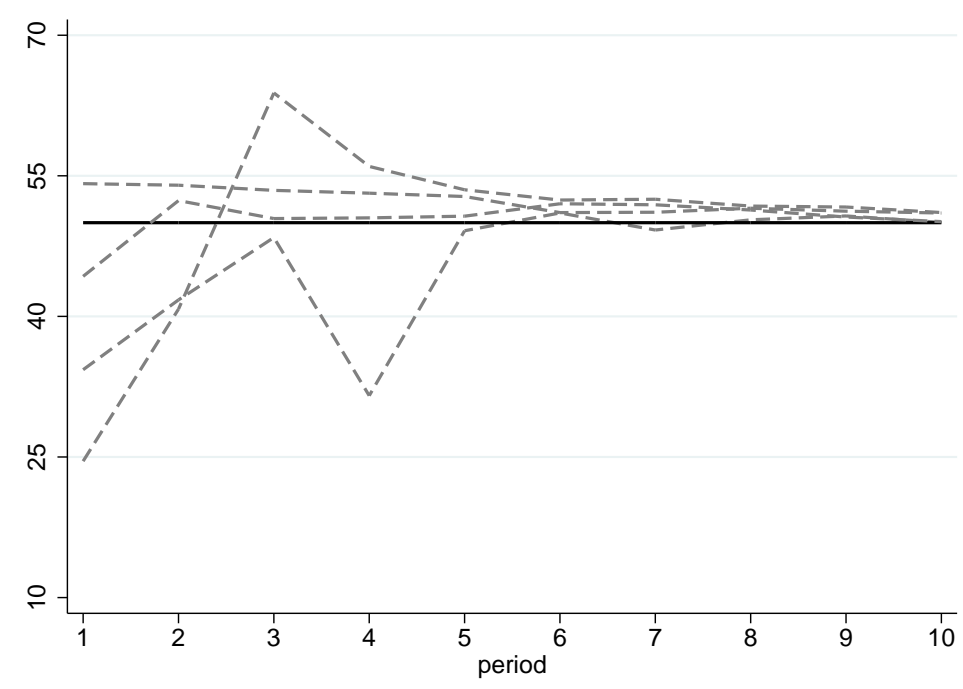

(c) 4 regular sessions: Anchor10

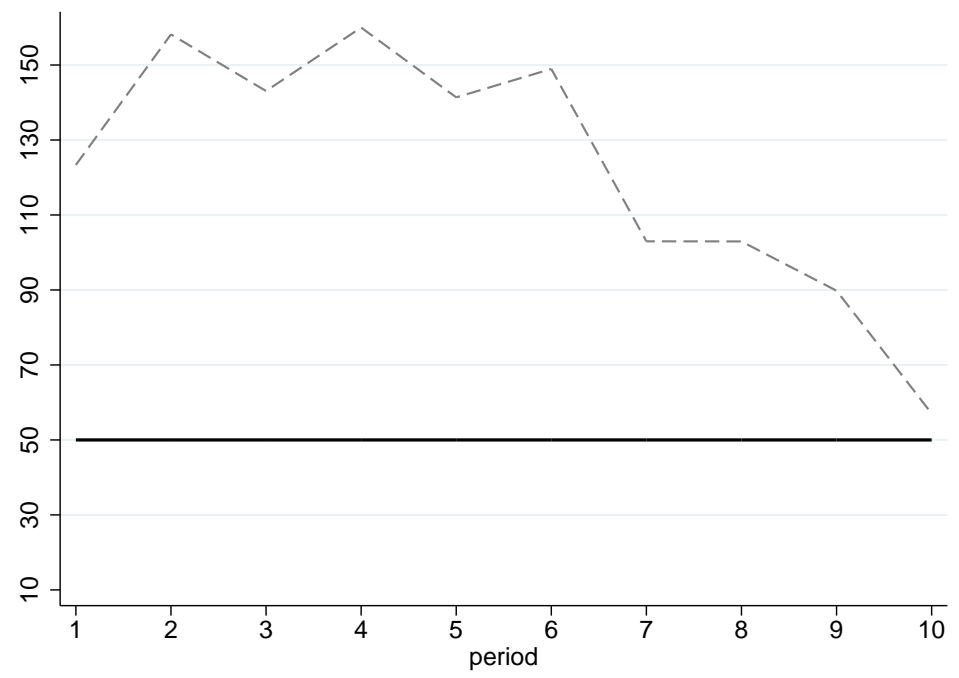

(b) 1 extreme session: Anchor90

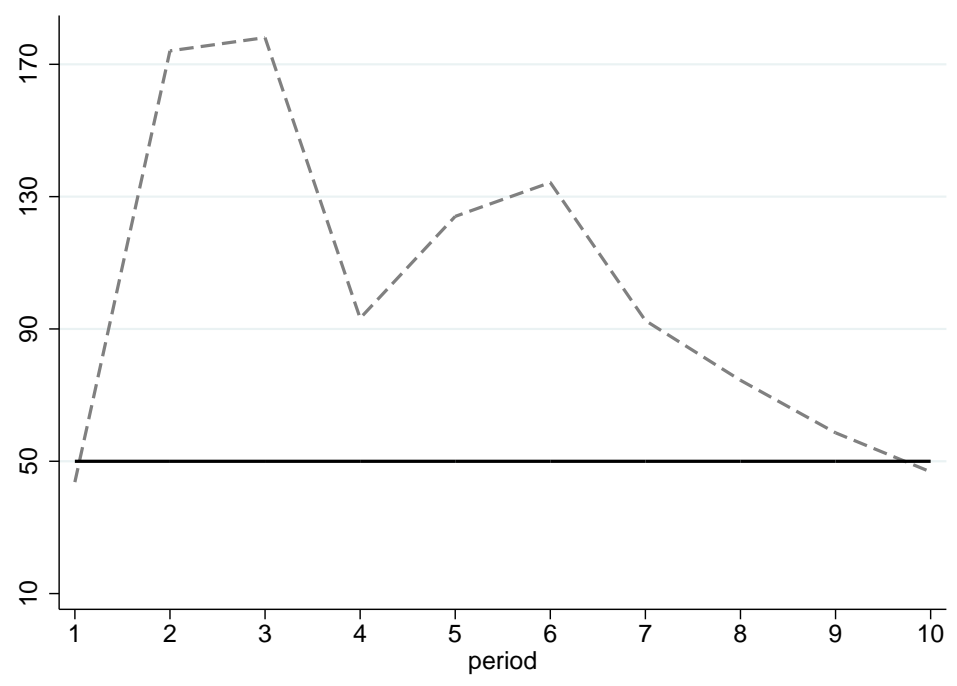

(d) 1 extreme session: Anchor10

Figure 8: Dashed Lines: Session-Prices. Solid Lines: FV (50). Figures 8a - 8b: Anchor at 90. Figures 8c - 8d: Anchor at 10. 


\section{Appendix D: The CPS Model}

In this section we show that our anchoring results can also be integrated into the model of Caginalp et al. [2000] (CPS hereafter), generating the observed asymmetric price patterns, by altering parameters which can be ascribed to initial anchoring. CPS present a model in which various variables, important for the formation of bubbles in experimental asset markets, can be summarized in the system of differential equations given in (6) - (10): ${ }^{26}$

$$
\begin{aligned}
& \frac{d}{d t} \log (p(t))=\log \frac{k(t)(1-B(t))}{(1-k(t)) B(t)} \\
& \frac{d}{d t} B(t)=k(1-B(t))-(1-k) B(t)+B(t)(1-B(t)) \frac{d}{d t} \log (p(t)) \\
& k(t)=\frac{1}{2}\left[1+\tanh \left(\xi_{1}(t)+\xi_{2}(t)\right)\right] \\
& \frac{d}{d t} \xi_{1}(t)=F_{1} \frac{d}{d t} \log (p(t)) \\
& \frac{d}{d t} \xi_{2}(t)=F_{2} \frac{F V-p(t)}{F V}
\end{aligned}
$$

where $p(t)$ denotes the price of the asset in period $t, B(t)$ is the fraction of total asset-value held in stocks, $k($.$) can be interpreted as the fraction of total cash used for the demand of assets, \xi_{1}(t)$ is the part of overall investor sentiment $\left(\xi_{1}(t)+\xi_{2}(t)\right)$ attributed to trend-sensitivity as a function of parameter $F_{1}$ and $\xi_{2}(t)$ is the part of overall investor sentiment measuring valuation-sensitivity as a function of parameter $F_{2}$. The mechanism through which the model of CPS generates bubbles is the following: If prices are initially below the $\mathrm{FV}$, traders increase their demand, resulting into positive price-momentum, since $\xi_{2}(t)>0$. When prices exceed the $\mathrm{FV}$, increased demand -which is sensitive to sentiment- already established a strong upward trend, generating an overshooting of prices through $\xi_{1}(t)$. The overvaluation turns into a crash when the effects of $\xi_{2}(t)$ dominate the effects of $\xi_{1}(t)$. The prices may then -in principle- undershoot the FV in this process, leading to price-oscillation around the FV with dampening amplitude. The magnitude of the over- and undershooting is governed by $F_{1}>0$ and $F_{2}>0$.

Note that both $F_{1}$ and $F_{2}$ are tightly related to the anchoring parameter in DÜ. For given $F_{2}$, the lower $F_{1}$, the more persistent are initial prices/anchors and the smaller the changes in behavior through actual changes in prices. Similarly, for given $F_{1}$, the lower $F_{2}$ the less sensitive are agents towards over- and undervaluation relative to the FV of the asset and deviations from the fundamental value may persist for extended periods of time. Since both parameters can be related

\footnotetext{
${ }^{26}$ Caginalp et al. [2000] who use this particular theoretical framework to explain price dynamics in experimental asset markets under initial price-restrictions. The authors test the main prediction of the model -that the size of the bubble is an increasing function of the initial undervaluation- by restricting initial prices to be below the FV of the asset. The theoretical framework offered in Caginalp et al. [2000], which relates initial conditions to subsequent price dynamics, also suits our purposes to investigate the effects of initial anchors on the observed price patterns.
} 
to anchoring we simplify the (already) parsimonious model of CPS further to avoid potential ambiguity in terms of identification. Precisely, we set $F_{1}=F_{2}=F$ and interpret a decrease in $F$ as an increase in anchoring towards initial prices.

To illustrate that the momentum-based model of CPS can generate asymmetric price-patterns, induced by anchoring, we solve the system of differential equations above with the initial conditions $B(0)=0.5$ and $\xi_{1}(0)=\xi_{2}(0)=0 .{ }^{27}$

In a first attempt we feed the system with the average initial prices from our "Anchor10$\mathrm{T} 4(\backslash=)$ " and our "Anchor90-T4 $(\backslash=)$ " treatments and fix F to a value of 0.7. The resulting price paths are shown in Figure 9a. Naturally, since the initial price in the "Anchor10-T4( $\backslash=)$ " treatment is below the FV we observe prices which increase initially, overshoot and finally converge towards the FV. The picture reverses for the "Anchor90-T4 $(\backslash=)$ " treatment. The high initial prices lead to an initial decrease of prices, followed by undershooting until prices eventually converge towards the FV. The degree of undershooting exceeds the degree of overshooting in the "Anchor10-T4 $(\backslash=)$ " treatment since initial prices are further away from the FV, generating a larger momentum effect. In contrast to the DÜ model the CPS model cannot generate asymmetric price paths by varying initial prices alone. However, given the initial conditions on the sentiment variables, the CPS model would generate the observation that prices stay at the FV if initiated there.

Next, we incorporate the findings from our DÜ model -which suggest that anchoring is less pronounced in our "Anchor10-T4 $(\backslash=)$ " treatment if compared to our "Anchor90-T4 $(\backslash=)$ " treatmentinto the CPS framework. More specifically, in addition to a change in prices we also change $F$ and set it equal to 0.7 for the "Anchor10-T4 $(\backslash=)$ " treatment and set it equal to 0.2 for the "Anchor90$\mathrm{T} 4(\backslash=)$ " treatment. In other words prices are more anchored towards initial conditions in the latter treatment than in the former.

The resulting price paths are shown in Figure 9b. We observe that the momentum-based model of CPS, after correcting for anchoring, generates (qualitatively) the asymmetries in the price paths we observe in our data. Intuitively, low initial prices induced via first period anchors, generate standard bubble-crash patterns via momentum. More specifically, initial undervaluation seems to make anchors less persistent, generating purchase decisions which drive up the price, sparking price-momentum. As prices increase, they may overshoot and peak out well above the asset's FV due to trend-sensitivity $\left(\xi_{1}\right)$ as a part of investor sentiment. As a result, valuation-sensitivity generates a downturn in prices.

Initial overvaluation, on the other hand -induced via visual stimulus-, seem to make anchors more persistent as was shown in the main body of the paper. In the model of CPS, this persistence can be parameterized via reduced trend- and valuation sensitivity, $F$. The resulting equilibrium price path converges slowly towards the FV from above, thereby generating price dynamics which

\footnotetext{
${ }^{27}$ Note that, evaluated at the fundamental value, half of the initial total asset holdings in our design are in the form of stocks. Hence a value of 0.5.
} 

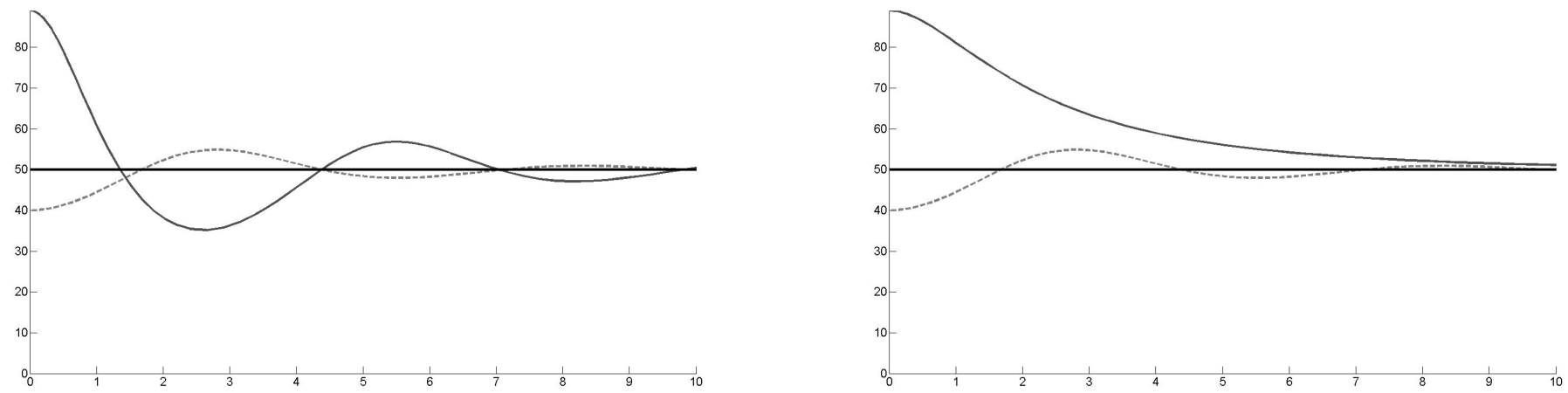

(a) Varying initial price in CPS. No change in the anchoring para-(b) Varying initial price in CPS and change in the anchoring parameter. meter.

Figure 9: Explaining price asymmetries. Dashed lines: Prices for low anchors. Solid lines (grey): Prices for high anchors. Solid horizontal lines (black): FV.

are consistent with our data. 


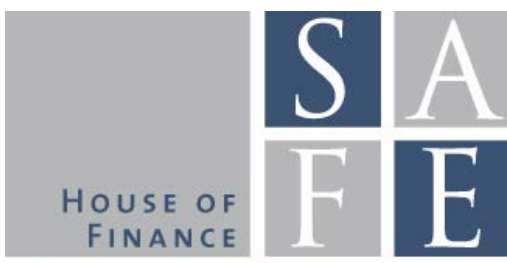

WORKING PAPER SERIES

\section{Recent Issues}

No. 53 Holger Kraft, Claus Munk, Frank Thomas Seifried, Morgens Steffensen

No. 52 Holger Kraft, Thomas Seiferling, Frank Thomas Seifried

No. 51 Yacine Aït-Sahalia, Roger J. A. Laeven, Loriana Pelizzon

No. 50 Ignazio Angeloni, Ester Faia, Roland Winkler

No.49 Gabriele Camera, Marco Casari, Stefania Bortolotti

No. 48 Marcel Bluhm, Jan Pieter Krahnen

No. 47 Michael Kosfeld, Ulrich Schüwer

No. 46 Marcel Bluhm, Ester Faia, Jan Pieter Krahnen

No. 45 Fabio Castiglionesi, Fabio Ferozzi, Gyongyi Loranth, Loriana Pelizzon

No. 44 Lorenz S. Schendel

No. 43 Lorenz S. Schendel
Consumption and Wage Humps in a LifeCycle Model with Education

Asset Pricing and Consumption-Portfolio Choice with Recursive Utility and Unspanned Risk

Mutual Excitation in Eurozone Sovereign CDS

Exit Strategies

An Experiment on Retail Payments Systems

Systemic Risk in an Interconnected Banking System with Endogenous Asset Markets

Add-On Pricing in Retail Financial Markets and the Fallacies of Consumer Education

Monetary Policy Implementation in an Interbank Network: Effects on Systemic Risk

Liquidity Coinsurance and Bank Capital

Critical Illness Insurance in Life Cycle Portfolio Problems

Consumption-Investment Problems with Stochastic Mortality Risk 